\title{
COHOMOLOGICAL APPROACH TO ASYMPTOTIC DIMENSION
}

\author{
A. Dranishnikov
}

\begin{abstract}
We introduce the notion of asymptotic cohomology based on the bounded cohomology and define cohomological asymptotic dimension $\operatorname{asdim}_{\mathbf{Z}} X$ of metric spaces. We show that it agrees with the asymptotic dimension $\operatorname{asdim} X$ when the later is finite. Then we use this fact to construct an example of a metric space $X$ of bounded geometry with finite asymptotic dimension for which $\operatorname{asdim}(X \times \mathbf{R})=\operatorname{asdim} X$. In particular, it follows for this example that the coarse asymptotic dimension defined by means of Roe's coarse cohomology is strictly less than its asymptotic dimension.
\end{abstract}

\section{$\S 0$ Introduction}

Gromov proposed to study discrete groups as large scale geometric objects. He introduced several asymptotic invariants of finitely generated groups [Gr1]. Among them there is the notion of asymptotic dimension which proved to be useful for the Novikovtype conjectures $[\mathrm{Yu}],[\mathrm{Ba}],[\mathrm{CG}],[\mathrm{Dr} 2],[\mathrm{DFW}]$. The asymptotic dimension differs from any other known dimension invariant of discrete groups. Thus, it differs from the geometric dimension $g d(\Gamma)[\mathrm{Br}]$ for every finite group $\Gamma$ since the geometric dimension is infinite for groups with torsions and the asymptotic dimension of finite groups is zero. If one takes into account only torsion free groups then the distinction between these two dimensions is not obvious. For almost all known torsion free groups where both invariants are computed there is the equality $\operatorname{asdim} \Gamma=g d(\Gamma)$. An exception is Gromov's example of a group $\Gamma_{0}$ with finite classifying space $B \Gamma_{0}$ that contains an expander (in some weakly coarse sense) [Gr2]. This group does not admit a coarse embedding into a Hilbert space and hence $[\mathrm{HR}]$, asdim $\Gamma_{0}=\infty$ whereas $g d\left(\Gamma_{0}\right)<\infty$.

1991 Mathematics Subject Classification. Primary 55M10, 20F69, 51F99, Secondary 57M20.

Key words and phrases. asymptotic dimension, bounded cohomology, coarse cohomology, asymptotic cohomological dimension, coarse cohomological dimension.

The author was partially supported by NSF grant DMS-061519

Typeset by $\mathcal{A} \mathcal{M S}-\mathrm{T}_{\mathrm{EX}}$ 
For discrete groups it is known that modulo some potential discrepancy in the case of geometric dimension three (Eilenberg-Ganea problem) there is the equality $g d(\Gamma)=c d(\Gamma)$ where $c d(\Gamma)$ is the cohomological dimension of $\Gamma$ with integral coefficients $[\mathrm{Br}]$. This equality gives an effective way for computation of $g d(\Gamma)$. A similar situation happens to be in the classical dimension theory of locally compact metric spaces. One of the basic facts there is the Alexandroff Theorem which states that the covering dimension agrees with the integral cohomological dimension: $\operatorname{dim} X=\operatorname{dim}_{\mathbf{Z}} X, \operatorname{provided} \operatorname{dim} X<\infty$. The cohomological approach in the classical dimension theory very often allows one to compute the covering dimension or to reduce the computation to a calculation of cohomological dimensions of $X$ with respect to the coefficient groups $\mathbf{Q}, \mathbf{Z}_{p}=\mathbf{Z} / p \mathbf{Z}$, and $\mathbf{Z}_{p^{\infty}}=\lim _{\rightarrow} \mathbf{Z}_{p^{k}}$ for prime $p$. Also it gives an exact formula for dimension of the product (Bockstein's formula [Dr3]). In particular the cohomological approach to dimension allows to prove Morita's theorem which states that $\operatorname{dim}(X \times \mathbf{R})=\operatorname{dim} X+1$ for general topological spaces $X$.

In the light of all this it would be useful to have a cohomological approach to the asymptotic dimension. The right approach would give answer to many naive still open questions in asymptotic dimension theory, like, Does the asymptotic Morita theorem hold true: $\operatorname{asdim}(\Gamma \times \mathbf{Z})=\operatorname{asdim} \Gamma+1$ for finitely generated groups $\Gamma$ ?

Perhaps the first attempt to define asymptotic dimension cohomologically was made in [Dr1] by means of Roe's coarse cohomology theory $H X^{*}$. We redefine the corresponding dimension in this paper $(\S 4)$ and call it the coarse cohomological dimension $\mathrm{X}-\operatorname{dim}_{G}$. It turns out that the coarse cohomological dimension $\mathrm{X}$ - $\operatorname{dim}_{G}$ does not always agree with the asymptotic dimension even for proper metric spaces of bounded geometry and when the later is finite $(\S 5)$. Thus, an asymptotic analog of Alexandroff Theorem does not hold for this dimension. We recall that the most elegant argument for the Alexandroff theorem uses the representation theorem for the Čech cohomology. In view of our result the macro-micro analogy suggests that Roe's coarse cohomology theory lacks Brown's representability.

Moreover, it turns out that the asymptotic Morita theorem does not hold true for general proper metric spaces of bounded geometry. We construct a counterexample in $\S 5$ based on a new cohomological approach to asymptotic dimension and an idea from elementary number theory. In $\S 6$ we show how this example can be turned into a large scale fractal-like space. We present there a general construction of large simplicial complexes of prescribed shapes on all scales.

A new cohomological approach to the asymptotic dimension is based on cohomology groups defined by bounded cochains. Using bounded cohomology we define an asymptotic cohomological dimension asdimz with integral coefficients in $\S 2$ and we show that $\operatorname{asdim}_{\mathbf{Z}} X=\operatorname{asdim} X$ for proper metric spaces of bounded geometry with asdim $X<\infty$ 
in $\S 3$. So this approach has an asymptotic analog of the Alexandroff Theorem and it also gives a hope for developing an analog of the Bockstein theory.

For finite coefficients $F$ the bounded cohomologies coincide with the standard cohomologies. We use this to show $(\S 4)$ that $\operatorname{asdim}_{F} Y=X-\operatorname{dim}_{F} Y$ in this case. In view of this equality, our approach to the asymptotic dimension still gives a hope for the formula $\operatorname{asdim} \Gamma=c d(\Gamma)$ for torsion-free finitely presented groups with finite asymptotic dimension. Now in order to derive this formula it suffices to show that in the case of a finitely presented group $\Gamma, \operatorname{asdim}_{\mathbf{Z}} \Gamma=\operatorname{asdim}_{\mathbf{Z}_{p}} \Gamma$ for some $p$. We recall that in the world of compacta this holds true for all locally nice spaces [Dr3]. It is still unclear whether finitely presented groups are sufficiently nice among all discrete proper metric spaces.

\section{$\S 1$ Preliminaries}

Asymptotic dimension. Gromov defined the asymptotic dimension of a metric space $X$ as follows [Gr1].

DEFINITION. A metric space $X$ has asymptotic dimension $\leq n$ if, for every $d>0$, there is an $R$ and $n+1 d$-disjoint, $R$-bounded families $\mathcal{U}_{0}, \mathcal{U}_{1}, \ldots, \mathcal{U}_{n}$ of subsets of $X$ such that $\cup_{i=0}^{n} \mathcal{U}_{i}$ is a cover of $X$.

We say that a family $\mathcal{U}$ of subsets of $X$ is $R$-bounded if $\sup \{\operatorname{diam} U \mid U \in \mathcal{U}\} \leq R$. Also, $\mathcal{U}$ is said to be $d$-disjoint if $d(x, y)>d$ whenever $x \in U, y \in V, U \in \mathcal{U}, V \in \mathcal{U}$, and $U \neq V$.

It is known that $\operatorname{asdim} X \leq n$ if and only if $X$ admits a uniformly bounded open cover $\mathcal{U}$ of multiplicity $\leq n+1$ with an arbitrary large Lebesgue number $L(\mathcal{U})=$ $\inf _{x \in X} \sup _{U \in \mathcal{U}} d(x, X \backslash U)$.

The notion of asymptotic dimension is a coarse invariant (see [Ro1] [Ro2]). Therefore the invariant asdim $\Gamma$ is well-defined for every finitely generated group (in fact for every countable group $\Gamma[\mathrm{DS}])$.

A metric space $X$ is called proper if every closed ball $B_{r}(x) \subset X$ is compact.

We recall that a cover $\mathcal{V}$ of a space $X$ is called a refinement of a cover $\mathcal{U}$, (the notation for this relation is $\mathcal{V} \prec \mathcal{U}$ ), if for every $V \in \mathcal{V}$ there is $U \in \mathcal{U}$ such that $V \subset U$. Note that if the mesh of $\mathcal{V}$ is less than the Lebesgue number $L(\mathcal{U})$ of $\mathcal{U}$, then $\mathcal{V} \prec \mathcal{U}$. A refinement defines a simplicial map of the nerves $q: N(\mathcal{V}) \rightarrow N(\mathcal{U})$ which is called a refinement map.

Let $\mathcal{C}$ be a collection of sets in $X$ and let $A \subset X$. The star of $A$ with respect to $\mathcal{C}$ is the set

$$
S t(A, \mathcal{C})=\bigcup_{C \in \mathcal{C}, C \cap A \neq \emptyset} C .
$$


If $\tau$ is the collection of simplices of a simplicial complex $K$, then for every vertex $v \in$ $K, S t(v, \tau)=S t(v, K)$ is the combinatorial star of the vertex. The open star of a vertex $O s t(v, K)=\operatorname{Int}(S t(v, K))$ can be also defined as the complement to the link $S t(v, K) \backslash L k(v, K)$.

A cover $\mathcal{V}$ of a space $X$ is called a star refinement of a cover $\mathcal{U}, \mathcal{V} \prec \prec \mathcal{U}$, if for every $V \in \mathcal{V}$ there is $U \in \mathcal{U}$ such that $S t(V, \mathcal{V}) \subset U$. The corresponding simplicial map $q: N(\mathcal{V}) \rightarrow N(\mathcal{U})$ is called a star refinement map.

Anti-Čech approximation. A sequence of uniformly bounded locally finite open coverings $\left\{\mathcal{U}_{i}\right\}$ of a metric space $X$ is called an anti-Čech approximation [Ro1] for $X$ if $\mathcal{U}_{i} \prec \mathcal{U}_{i+1}$ for all $i$ and the Lebesgue number $L\left(\mathcal{U}_{i}\right)$ tends to infinity. Let $N_{i}=N\left(\mathcal{U}_{i}\right)$ denote the nerve of $\mathcal{U}_{i}$. Then every anti-Čech approximation defines a direct system of locally finite simplicial complexes with refinement maps as the bonding maps:

$$
N_{1} \stackrel{q_{2}^{1}}{\longrightarrow} N_{2} \stackrel{q_{3}^{2}}{\longrightarrow} \ldots \rightarrow N_{k} \stackrel{q_{k+1}^{k}}{\longrightarrow} N_{k+1} \rightarrow \ldots
$$

For a locally finite open cover $\mathcal{U}$ of a metric space $X$ let $p: X \rightarrow N(\mathcal{U})$ denote a projection to the nerve defined by the partition of unity $\left\{\phi_{U}\right\}$ with $\phi_{U}^{-1}(0)=X \backslash U$. We call such projection canonical if it is defined by the following partition of unity:

$$
\phi_{U}(x)=\frac{d(x, X \backslash U)}{\sum_{V} d(x, X \backslash V)} .
$$

We recall that a cover $\mathcal{U}$ is called irreducible if for every $U \in \mathcal{U}$ the family $\mathcal{U} \backslash\{U\}$ is not a cover. If the cover $\mathcal{U}$ is irreducible then all vertices of $N(\mathcal{U})$ are in the image of $p$. For a map $p$ with such property we say that it is essentially surjective. We always will assume that the covers $\mathcal{U}_{i}$ in the definition of an anti-Čech approximation are irreducible.

A uniform metric on a simplicial complex $K$ is the metric restricted from the Hilbert space $\ell_{2}\left(K^{(0)}\right)$ under the natural realization of $K$. The geodesic metric on $K$ induced from the uniform metric is called uniform geodesic. Usually we normalize this metric by $1 / \sqrt{2}$ to have the length of every edge in all simplices equal one.

A map $f: X \rightarrow K$ of a metric space to a simplicial complex is called uniformly cobounded if there is $D>0$ such that $\operatorname{diam}\left(f^{-1}(\Delta)\right) \leq D$ for every simplex $\Delta$.

Let $f: X \rightarrow Y$ be a map between metric spaces. We denote the number (if it exists)

$$
\operatorname{Lip}(f)=\sup \left\{\frac{d_{Y}(f(x), f(y))}{d_{X}(x, y)} \mid x, y \in X, x \neq y\right\}
$$

and call it the Lipschitz constant of $f$. Every number $\lambda \geq \operatorname{Lip}(f)$ is called a Lipschitz constant for $f$. 
We note that for every irreducible uniformly bounded locally finite open cover $\mathcal{U}$ of a metric space $X$ with the Lebesgue number $L$ the canonical projection $p: X \rightarrow N(\mathcal{U})$ to the nerve is $c_{n} / L$-Lipschitz and uniformly cobounded where $n$ is the multiplicity of $\mathcal{U}$ [BD1]. Moreover, it is a quasi-isometry provided $X$ is geodesic and the nerve is taken with geodesic metric:

We recall that $p: X \rightarrow Y$ is a $(l, D)$-quasi-isometric embedding if there are constants $\lambda \geq 1$ and $D$ such that

$$
\frac{1}{l} d_{X}(x, y)-D \leq d_{Y}(p(x), p(y)) \leq l d_{X}(x, y)+D .
$$

An $(l, D)$-quasi-isometric embedding $p: X \rightarrow Y$ is called a quasi-isometry if there is a constant $r$ such that the image $p(X)$ is $r$-dense in $Y$, i.e. $N_{r}(p(X))=Y$. We use the notations $N_{r}(A)=\{x \in X \mid d(x, A) \leq r\}$ and $O N_{r}(A)=\{x \in Y \mid d(x, A)<r\}, r>0$ for closed and open $r$-neighborhoods of the set $A$ in a metric space $Y$. Also we consider "negative" neighborhoods $N_{-r}(A)=Y \backslash N_{r}(X \backslash A), r>0$.

The following lemma can be extracted from [BD1] and Dr4].

Lemma 1.1. Given $n$ there is a constant $C_{n}$ such that for every uniformly bounded cover $\mathcal{U}$ of a proper geodesic metric space $X$ with multiplicity of $\mathcal{U} \leq n+1$ the canonical projection $p: X \rightarrow N(\mathcal{U})_{U G}$ to the nerve is a $\epsilon$-Lipschitz $(l, 3)$-quasi-isometry with $r=1$ where $\epsilon=C_{n} / L(\mathcal{U})$ and $l=C_{n}$ mesh $(\mathcal{U})$.

Note that if the multiplicity of coverings $\mathcal{U}_{i}$ in an anti-Čech approximation of $X$ is bounded from above then the canonical projections $p_{i}: X \rightarrow N\left(\mathcal{U}_{i}\right)$ are $\epsilon_{i}$-Lipschitz with $\lim \epsilon_{i}=0$. Thus, a metric space $X$ has $\operatorname{asdim} X \leq n$ if and only if it admits an anti-Čech approximation $\mathcal{U}_{1} \prec \mathcal{U}_{2} \prec \mathcal{U}_{3} \prec \ldots$ with the multiplicity of $\mathcal{U}_{i}$ bounded from above by $n+1$. In terms of projections to the nerves it equals to the property that $X$ admits a sequence $p_{i}: X \rightarrow N_{i}$ of uniformly cobounded $\epsilon_{i}$-Lipschitz maps to uniform $n$-dimensional complexes with $\lim \epsilon_{i}=0$.

We recall that a metric space $X$ is said to be of bounded geometry (on large scale) if for every $R$ the 1-capacity of $R$-balls in $X$ is uniformly bounded from above. It was shown in [Ro1] that given $\lambda>0$, every metric space of bounded geometry $X$ admits an open cover of finite multiplicity with the Lebesgue number greater than $\lambda$. Thus every metric space of bounded geometry admits an anti-Čech approximation.

Let $p: K \rightarrow N$ be map between simplicial complexes. A simplicial map $q: K \rightarrow N$ is called a simplicial approximation of $p$ if $p^{-1}(\Delta) \subset q^{-1}(\Delta)$ for every simplex $\Delta \subset N$. This is equivalent to the condition $p^{-1}(L) \subset q^{-1}(L)$ for every subcomplex $L \subset N$. 
Proposition 1.2. Every proper geodesic metric space $X$ with asdim $X \leq n$ admits an anti-Čech approximation $\left\{\mathcal{U}_{i}, q_{i+1}^{i}\right\}$ with $n$-dimensional locally finite nerves $N_{i}$ and essentially surjective projections $p_{i}: X \rightarrow N_{i}$ such that

(1) there are bonding maps $p_{i+1}^{i}: N_{i} \rightarrow N_{i+1}$ with $p_{i+1}=p_{i+1}^{i} \circ p_{i}$ for all $i$,

(2) $\left(p_{i+1}^{i}\right)^{-1}(K)$ is a subcomplex for every subcomplex $K \subset N_{i+1}$,

(3) simplicial maps $q_{i+1}^{i}: N_{i} \rightarrow N_{i+1}$ are simplicial approximations of $p_{i+1}^{i}$,

(4) $\operatorname{Lip}\left(p_{i+1}^{i}\right)<1 / 2$, and

(5) $\mathcal{U}_{i} \prec \prec \mathcal{U}_{i+1}$ for all $i$ and $q_{i+1}^{i}$ is a star refinement map.

Proof. We construct these coverings and maps by induction. Assume that a sequence of covers $\mathcal{U}_{1} \prec \prec \mathcal{U}_{2} \prec \prec \ldots \mathcal{U}_{k}$ together with the maps $p_{i}, p_{i+1}^{i}$, and $q_{i+1}^{i}$ satisfying conditions (1)-(5) is constructed. We assume that $p_{1}$ and all $p_{i+1}^{i}$ are canonical projections to the nerves. By Proposition $1.1 N_{k}$ is quasi-isometric to $X$ and hence asdim $N_{k} \leq n$. We consider a uniformly bounded cover $\mathcal{V}^{\prime}$ of $N_{k}$ of multiplicity $n+1$ with the Lebesgue number $\geq 2 C_{n}+2 \geq 5$. Then $N_{-1}\left(\mathcal{V}^{\prime}\right)=\left\{N_{-1}\left(V^{\prime}\right) \mid V^{\prime} \in \mathcal{V}^{\prime}\right\}$ has the Lebesgue number $\geq 2 C_{n}+3$. We define $\mathcal{V}=\left\{\operatorname{Int}\left(\operatorname{St}\left(V, N_{k}\right)\right) \mid V \in N_{-1}\left(\mathcal{V}^{\prime}\right)\right\}$. Then $\mathcal{V} \prec \mathcal{V}^{\prime}$ and hence, the nerve of $\mathcal{V}$ is $n$-dimensional and $\left\{\operatorname{Ost}\left(v, N_{k}\right) \mid v \in N_{k}^{(0)}\right\} \prec \prec \mathcal{V}$. Define $\mathcal{U}_{k+1}=p_{k}^{-1} \mathcal{V}$. Then

$$
\mathcal{U}_{k}=p_{k}^{-1}\left\{O s t\left(v, N_{k}\right) \mid v \in N_{k}^{(0)}\right\} \prec \prec p_{k}^{-1} \mathcal{V}=\mathcal{U}_{k+1} .
$$

Let $N_{k+1}=N(\mathcal{V})=N\left(\mathcal{U}_{k+1}\right)$, let $p_{k+1}^{k}: N_{k} \rightarrow N_{k+1}$ be the canonical projection to the nerve, and let $p_{k+1}=p_{k+1}^{k} p_{k}$. We define $q_{k+1}^{k}(v)$ to be an element $\operatorname{Int}\left(\operatorname{St}\left(V, N_{k}\right)\right)$ of $\mathcal{V}, V \in \mathcal{V}^{\prime}$ such that $S t(v) \subset V$. By Proposition $1.2 p_{k+1}^{k}$ is $C_{n} / L(\mathcal{V})$-Lipschitz. Thus, $\operatorname{Lip}\left(p_{k+1}^{k}\right) \leq 1 / 2$ by the choice of $\mathcal{V}$. Thus the conditions (1),(4), and (5) are satisfied.

To verify (2) it suffices to show that the preimage $\left(p_{k+1}^{k}\right)^{-1}(\Delta)$ is a subcomplex in $N_{k}$ for every simplex $\Delta$ in $N(\mathcal{V})$. Note that

$$
\left(p_{k+1}^{k}\right)^{-1}(\Delta)=N_{k} \backslash \bigcup_{W \notin \Delta(0)} W=N_{k} \backslash \bigcup_{W} \operatorname{Int}\left(K_{W}\right)=\bigcap_{W}\left(N_{k} \backslash \operatorname{Int} K_{W}\right)
$$

where $K_{W}=S t\left(V_{W}, N_{k}\right)$ is a subcomplex of $N_{k}$. Hence $\left(p_{k+1}^{k}\right)^{-1}(\Delta)$ is a subcomplex of $N_{k}$ as the intersection of subcomplexes.

Finally we check (3). Let $p_{k+1}^{k}(z) \in \Delta=\left[V_{1}, \ldots, V_{j}\right]$. Then if $z \in V \in \mathcal{V}$, it follows that $V$ equals one of $V_{i}, i \leq j$. Let $z \in\left[v_{1}, \ldots, v_{s}\right] \subset N_{k}$. By the definition $z \in S t\left(v_{i}\right) \subset q_{k+1}^{k}\left(v_{i}\right)$. Therefore, $q_{k+1}^{k}\left(v_{i}\right) \in \Delta^{(0)}$. Hence $q_{k+1}^{k}(z) \in \Delta$.

We will refer to such anti-Čech approximation as to regular and will denote it as

$$
\left\{p_{i}: X \rightarrow N_{i}, q_{i+1}^{i}, p_{i+1}^{i}\right\}
$$


where $p_{i}$ are $\epsilon_{i}$-Lipschitz with $\lim \epsilon_{i}=0$. In some instances we will not assume the condition (5). Note that the coverings $\mathcal{U}_{i}$ can be recovered from this data as $\mathcal{U}_{i}=$ $p_{i}^{-1}\left(\operatorname{Ost}\left(v, N_{i}\right)\right)$ where $\operatorname{Ost}(v, N)$ is the open star of a vertex $v$ in a simplicial complex $N$.

\section{$\S 2$ Definition of asdimz}

Bounded cohomology. Let $K$ be a simplicial complex. An integral cochain $\phi$ : $C_{m}(K) \rightarrow \mathbf{Z}$ is bounded if there is a constant $b<\infty$ such that $|\phi(\sigma)|<b$ for all $m$-simplices $\sigma$ in $K$. Clearly, the coboundary $\delta \phi$ of a bounded cochain is a bounded cochain. Cohomology groups defined by means of bounded cochains $C_{b}^{*}$ are called bounded cohomologies of $K$ and denoted as $H_{b}^{*}(K)$. Clearly, the inclusion $C_{b}^{*} \rightarrow C^{*}$ is a chain map. Therefore there is a natural homomorphism $H_{b}^{*}(K) \rightarrow H^{*}(K)$. Every simplicial map $\phi: K \rightarrow L$ induces a homomorphism $H_{b}^{*}(L) \rightarrow H_{b}^{*}(K)$. This definition can be extended to any coefficient group with a norm such as $\mathbf{Q}$ or $\mathbf{R}$ and their subgroups. For a subcomplex $L \subset K$ one can define a relative bounded cohomology groups $H_{b}^{*}(K, L)$ by

considering the relative cochains. We note that for bounded cohomology there are the exact sequence of pair

$$
\ldots \leftarrow H_{b}^{i}(L) \leftarrow H_{b}^{i}(K) \leftarrow H_{b}^{i}(K, L) \leftarrow H_{b}^{i-1}(L) \leftarrow \ldots,
$$

exact sequence of triple

$$
\ldots \leftarrow H_{b}^{i}(L, A) \leftarrow H_{b}^{i}(K, A) \leftarrow H_{b}^{i}(K, L) \leftarrow H_{b}^{i-1}(L, A) \leftarrow \ldots
$$

where $A \subset L$ is a subcomplex, and the excision isomorphism

$$
H_{b}^{i}(K, B) \stackrel{\cong}{\rightrightarrows} H_{b}^{i}(A, A \cap B)
$$

where $A, B \subset K$ are subcomplexes such that $K=A \cup B$.

Approximation by asymptotic polyhedra. Let $X$ be a metric space. We consider locally finite covers $\mathcal{U}$ of $X$ by bounded open sets such that the Lebesgue number $L_{x}(\mathcal{U})$ tends to infinity as $x \rightarrow \infty$. We say that $\mathcal{V}$ is a refinement of $\mathcal{U}$ at infinity if there is $R>0$ such that for every $V \in \mathcal{V}, d\left(V, x_{0}\right)>R$, there is $U \in \mathcal{U}$ with $V \subset U$. A refinement at infinity $\mathcal{V} \prec_{\infty} \mathcal{U}$ defines a simplicial map $\phi: K_{\mathcal{V}} \rightarrow K_{\mathcal{U}}$ between corresponding subcomplexes of the nerves. All such covers with the relation $\prec_{\infty}$ form a directed set $a \operatorname{Cov}(X)$.

The following proposition is proven in [Dr1]. 
Proposition 2.1. If a metric space $X$ has asdim $X \leq n$ then the family aCov $(X)$ has a cofinal subfamily aCov $(X)$ that consists of covers with $n$-dimensional nerves.

A countable simplicial complex $K$ with a metric $d$ such that every simplex is isometric to an affine simplex in a Hilbert space is called an asymptotic polyhedron if $\lim _{i \rightarrow \infty} \operatorname{Lip}\left(\phi_{i}\right)=0$ where $K$ is the union of simplices $K=\cup_{i} \sigma_{i}$ and $\phi_{i}: \sigma_{i} \rightarrow \Delta^{\operatorname{dim} \sigma_{i}}$ is the affine map to the standard simplex.

We note that if a cover $\mathcal{U}$ has bounded multiplicity then its nerve $N$ admits a metric of an asymptotic polyhedron such that the projection $p_{\mathcal{U}}: X \rightarrow N$ is 1-Lipschitz (see [Dr1] [DFW]).

Asymptotic bounded cohomology. Let $K$ be a simplicial complex. Denote by $\mathcal{F}(K)$ the set of all finite subcomplexes of $K$. We define the bounded cohomology at infinity $A H_{b}^{i}(K)$ of a complex $K$ in the dimension $i$ as the direct limit

$$
A H_{b}^{i}(K)=\lim _{\rightarrow}\left\{H_{b}^{i}(K \backslash F) \mid F \subset K, F \in \mathcal{F}(K)\right\}
$$

Let $X$ be a metric space and let $\mathcal{V} \prec_{\infty} \mathcal{U}$ be covers as above. Then there is a welldefined homomorphism $A H_{b}^{*}(N(\mathcal{U})) \rightarrow A H_{b}^{*}(N(\mathcal{V}))$ between the bounded cohomology at infinity of nerves.

For a metric space $X$ we define its asymptotic bounded cohomology as the direct limit

$$
A H^{i}(X)=\lim _{\rightarrow}\left\{A H_{b}^{i}(N(\mathcal{U})) \mid \mathcal{U} \in a \operatorname{Cov}(X)\right\}
$$

Thus, it can be defined as

$$
A H^{i}(X)=\lim _{\rightarrow}\left\{H_{b}^{i}(N(\mathcal{U}) \backslash F) \mid \mathcal{U} \in a \operatorname{Cov}(X), F \in \mathcal{F}(N(\mathcal{U}))\right\}
$$

This definition can be extended to any coefficient group with the norm.

Let $L$ be a subcomplex of a simplicial complex $K$. We define the bounded cohomology at infinity $A H_{b}^{*}(K, L)$ of the pair $(K, L)$ in the dimension $i$ as the direct limit

$$
A H_{b}^{i}(K, L)=\lim _{\rightarrow}\left\{H_{b}^{i}(K \backslash F, L \backslash F) \mid F \subset K, F \in \mathcal{F}\right\}
$$

Let $X$ be a metric space and let $Y \subset X$ be a subset. For a cover $\mathcal{U}$ of $X$ we denote by $N\left(\left.\mathcal{U}\right|_{Y}\right)$ the nerve of the cover $\mathcal{U}$ restricted to $Y,\left.\mathcal{U}\right|_{Y}=\{U \cap Y \mid U \in \mathcal{U}\}$. Clearly, $N\left(\left.\mathcal{U}\right|_{Y}\right) \subset N(\mathcal{U})$ and a refinement $\mathcal{V} \prec \mathcal{U}$ defines a simplicial map of pairs $\left(N(\mathcal{V}), N\left(\left.\mathcal{V}\right|_{Y}\right)\right) \rightarrow\left(N(\mathcal{V}), N\left(\left.\mathcal{U}\right|_{Y}\right)\right)$. Then we define a relative asymptotic cohomology as the direct limit

$$
A H^{i}(X, Y)=\lim _{\rightarrow}\left\{A H_{b}^{i}\left(N(\mathcal{U}), N\left(\left.\mathcal{U}\right|_{Y}\right)\right) \mid \mathcal{U} \in a \operatorname{Cov}(X)\right\}
$$


Let $Y \subset X$ be a subset of a metric space. For a cover $\mathcal{U}$ of $X$ we denote by $\mathcal{U}_{Y}=$ $\{U \in \mathcal{U} \mid U \cap Y \neq \emptyset\}$. By $a \operatorname{Cov}_{X}(Y)$ we denote the set of locally finite covers $\mathcal{V}$ of $Y$ by bounded open sets from $X$ with $\lim _{x \rightarrow \infty, x \in Y} L_{x}(\mathcal{V})=\infty$. Thus, for every $\mathcal{U} \in a \operatorname{Cov}(X)$ we have $\mathcal{U}_{Y} \in a \operatorname{Cov}_{X}(Y)$. Note that every cover $\mathcal{V} \in a \operatorname{Cov}_{X}(Y)$ can be enlarge to a cover $\mathcal{U} \in a \operatorname{Cov}(X), \mathcal{V} \subset \mathcal{U}$, in such a way that $\left.\mathcal{U}\right|_{Y}=\left.\mathcal{V}\right|_{V}$.

Proposition 2.2. The family $\left\{\left.\mathcal{U}\right|_{Y} \mid \mathcal{U} \in a \operatorname{Cov}(X)\right\}=\left\{\left.\mathcal{U}\right|_{Y} \mid \mathcal{U} \in a \operatorname{Cov}_{X}(Y)\right\}$ is cofinal in aCov $(Y)$ where $Y \subset X$ is taken with the restriction metric.

Proof. Let $\mathcal{W} \in a \operatorname{Cov}(Y)$. For every $W \in \mathcal{W}$ we define $\tilde{W}=\cup_{x \in W} B_{r_{x} / 3}(x)$ where $r_{x}$ is the supremum of $r$ such that $B_{r}(x) \cap Y \subset W$ and $B_{r}(x)$ is the $r$-ball in $X$. Clearly, $\tilde{W} \cap Y=W$. Let $\tilde{\mathcal{W}}=\{\tilde{W} \mid W \in \mathcal{W}\}$. We note the Lebesgue number of $\tilde{\mathcal{W}}$ restricted to $Y$ tends to infinity. Thus, $\tilde{W} \in a \operatorname{Cov}_{X}(Y)$

REMARK. Note that $N(\tilde{\mathcal{W}})=N(\mathcal{W})$. Since $\{\tilde{\mathcal{W}} \mid \mathcal{W} \in \operatorname{aCov}(Y)\}$ is cofinal in $a_{\operatorname{Cov}_{X}}(Y)$, we obtain

$$
A H^{*}(Y)=\lim _{\rightarrow}\left\{A H_{b}^{*}\left(N\left(\left.\mathcal{U}\right|_{Y}\right)\right) ; \mathcal{U} \in a \operatorname{Cov}(X)\right\}=\lim _{\rightarrow}\left\{A H_{b}^{*}\left(N\left(\mathcal{U}_{Y}\right)\right) ; \mathcal{U} \in a \operatorname{Cov}(Y)\right\} .
$$

Thus, the asymptotic cohomology of a pair can be defined as

$$
A H^{i}(X, Y)=\lim _{\rightarrow}\left\{A H_{b}^{i}\left(N(\mathcal{U}), N\left(\mathcal{U}_{Y}\right)\right) \mid \mathcal{U} \in a \operatorname{Cov}(X)\right\} .
$$

Since bounded cohomologies at infinity posses the exactness, the exactness is preserved by direct limits, and in view of Proposition 2.2, there are exact sequences for asymptotic cohomology of pair (and triple).

A triad $X, A, B, A \cup B=X$, is called excisable if the family $\left\{\mathcal{U}_{A} \cap \mathcal{U}_{B} \mid \mathcal{U} \in a \operatorname{Cov}(X)\right\}$ is cofinal in $a \operatorname{Cov}_{X}(A \cap B)$.

Proposition 2.3. For every excisable triad $X, A, B$ there is the Mayer-Vietoris exact sequence

$$
\cdots \rightarrow A H^{n}(A) \oplus A H^{n}(B) \rightarrow A H^{n}(X) \rightarrow A H^{n+1}(A \cap B) \rightarrow \ldots
$$

Proof. We note that the Mayer-Vietoris sequence holds for bounded cohomology and hence for bounded cohomology at infinity for simplicial complexes. We consider the direct limit of these Mayer-Vietoris sequences for the nerves $N(\mathcal{U}), \mathcal{U} \in a \operatorname{Cov}(X)$ :

$$
\rightarrow A H_{b}^{n}\left(N\left(\mathcal{U}_{A}\right)\right) \oplus A H_{b}^{n}\left(N\left(\mathcal{U}_{B}\right)\right) \rightarrow A H_{b}^{n}(N(\mathcal{U})) \rightarrow A H_{b}^{n+1}\left(N\left(\mathcal{U}_{A}\right) \cap N\left(\mathcal{U}_{B}\right)\right) \rightarrow
$$


Note that there are the inclusions $N\left(\mathcal{U}_{A}\right) \cap N\left(\mathcal{U}_{B}\right)=N\left(\mathcal{U}_{A} \cap \mathcal{U}_{B}\right)$. Since $\left\{\mathcal{U}_{A} \cap \mathcal{U}_{B} \mid \mathcal{U} \in\right.$ $a \operatorname{Cov}(X)\}$ is cofinal in $a \operatorname{Cov}_{X}(A \cap B)$, one can argue that

$$
\lim _{\rightarrow} A H_{b}^{*}\left(\left(N\left(\left.\mathcal{U}\right|_{A}\right) \cap N\left(\left.\mathcal{U}\right|_{B}\right)\right)=\lim _{\rightarrow} A H_{b}^{*}\left(N\left(\left.\mathcal{U}\right|_{A \cap B}\right)\right)=A H^{*}(A \cap B)\right.
$$

There is a version of the Mayer-Vietoris sequence for pairs.

Proposition 2.4. For every excisable triad $A \cup B, A, B$ of subsets in a metric space $X$ there is the Mayer-Vietoris exact sequence

$$
\cdots \rightarrow A H^{n}(X, A) \oplus A H^{n}(X, B) \rightarrow A H^{n}(X, A \cap B) \rightarrow A H^{n+1}(X, A \cup B) \rightarrow \ldots
$$

A metric space $X$ is called uniformly path connected if there is a monotone tending to infinity continuous function $S: \mathbf{R}_{+} \rightarrow \mathbf{R}_{+}, S(0)=0$, such that every two points $x, x^{\prime} \in X$ can be joined by a path $J$ with $\operatorname{diam}(J) \leq S\left(d\left(x, x^{\prime}\right)\right)$. We note that every geodesic metric space is uniformly path connected.

Proposition 2.5. Let $W \subset X$ be an open subset of a uniformly path connected metric space $X$. Then the triple $X, \bar{W}, X \backslash W$ is excisable.

Proof. Let $\mathcal{V} \in a \operatorname{Cov}_{X} \partial W$. Let $\mathcal{U}^{\prime} \in a \operatorname{Cov}(X)$ be an enlargement of $\mathcal{V}, \mathcal{V} \subset \mathcal{U}^{\prime}$, and $\left.\mathcal{U}^{\prime}\right|_{Y}=\left.\mathcal{V}\right|_{Y}$. Since $X$ is uniformly path connected, the cover $\mathcal{U}$ that consists of components of sets from $\mathcal{U}^{\prime}$ belongs to $a \operatorname{Cov}(X)$. Then for every $U \in \mathcal{U}$ with $U \cap \bar{W} \neq \emptyset$ and $U \cap(X \backslash W) \neq \emptyset$, we obtain $U \cap \partial W \neq \emptyset$. Thus, $U$ is a component of $U^{\prime}$ with $U^{\prime} \in \mathcal{V}$. We have checked that $\left.\left.\mathcal{U}\right|_{\bar{W}} \cap \mathcal{U}\right|_{X \backslash W} \prec \mathcal{V}$.

Proposition 2.6. Let $W \subset X$ be an open subset of a uniformly path connected metric space $X$. Then there is the excision isomorphism

$$
A H^{n}(X, X \backslash W)=A H^{n}(\bar{W}, \partial W)
$$

Proof. Let $\mathcal{U} \in a \operatorname{Cov}(X)$. Note that there is an inclusion $\partial N\left(\mathcal{U}_{\bar{W}}\right) \subset N\left(\mathcal{U}_{\bar{W}} \cap \mathcal{U}_{X \backslash W}\right)$ which is essentially onto. Moreover, if $\mathcal{V} \prec \prec \mathcal{U}$, then there is a map $\xi: N\left(\mathcal{V}_{\bar{W}} \cap \mathcal{V}_{X \backslash W}\right) \rightarrow$ $\partial N\left(\mathcal{U}_{\bar{W}}\right)$ that make the diagram generated by $\xi$ and the refinement map commutative. This implies that

$$
\lim _{\rightarrow} A H_{b}^{*}\left(\partial N\left(\mathcal{U}_{\bar{W}}\right)\right) \rightarrow \lim _{\rightarrow} A H_{b}^{*}\left(N\left(\mathcal{U}_{\bar{W}} \cap \mathcal{U}_{X \backslash W}\right)\right)
$$


is an isomorphism. In view of Proposition 2.5 the later limit is equal to $\lim _{\rightarrow} A H_{b}^{*}\left(N\left(\mathcal{U}_{\partial W}\right)\right)$. Therefore,

$$
\lim _{\rightarrow} A H_{b}^{*}\left(N\left(\mathcal{U}_{\bar{W}}\right), \partial N\left(\mathcal{U}_{\bar{W}}\right)\right)=\lim _{\rightarrow} A H_{b}^{*}\left(N\left(\mathcal{U}_{\bar{W}}\right), N\left(\mathcal{U}_{\partial W}\right)\right)=A H^{*}(\bar{W}, \partial W) .
$$

By the excision for bounded cohomology at infinity of simplicial complexes we obtain

$$
A H^{*}(X, X \backslash W)=\lim _{\rightarrow} A H_{b}^{*}\left(N(\mathcal{U}), N\left(\mathcal{U}_{X \backslash W}\right)=\lim _{\rightarrow} A H_{b}^{*}\left(N\left(\mathcal{U}_{\bar{W}}\right), \partial N\left(\mathcal{U}_{\bar{W}}\right)\right) .\right.
$$

Asymptotic cohomological dimension. Let $X$ be a metric space we define its asymptotic integral cohomological dimension as follows:

$$
\operatorname{asdim}_{\mathbf{Z}} X=\max \left\{n\left|A H^{n}(A, B) \neq 0\right| B \subset A \subset X\right\} .
$$

This definition can be extended to any coefficient group $G$ with a semi-norm. The notation is $\operatorname{asdim}_{G} X$ when the semi-norm on $G$ is specified.

REMARK. One can show that like in the case of cohomological dimension in topology it suffices to consider $A=X$ in the above definition. Indeed, from exact sequence of triple $B \subset A \subset X$

$$
A H^{n+1}(X, A) \leftarrow A H^{n}(A, B) \leftarrow A H^{n}(X, B) \leftarrow A H^{n}(X, A) \leftarrow
$$

it follows that if $A H^{n}(A, B) \neq 0$ for $n=\operatorname{asdim}_{\mathbf{Z}} X$, then $A H^{n+1}(X, A)=0$ and hence $A H^{n}(X, B) \neq 0$.

\section{$\S 3$ Connection Between asdim $\mathbf{z}$ And asdim}

In this section we show that asdimz agrees with asdim for proper geodesic metric spaces provided the later is finite.

Proposition 3.1. Let $K \subset N$ be a subcomplex of a uniform geodesic complex of dimension n. Suppose that $f: K \rightarrow L$ is the map to an b-bounded metric space such that Lip $\left(\left.f\right|_{\sigma}\right) \leq \lambda, \lambda \geq 1$, for all simplices $\sigma \subset K$. Then $f$ is $c \lambda$-Lipschitz where $c$ depends on $n$ and $b$ only.

Proof. Let $K$ be realized in the Hilbert space $\ell_{2}\left(K^{(0)}\right)$ spanned by the vertices of $K$. Then the identity map $1_{K}:\left(K, d_{N}\right) \rightarrow\left(K, d_{\ell_{2}}\right)$ is 1-Lipschitz. It suffices to show that $f$ 
is $c \lambda$-Lipschitz with respect to the Hilbert space metric. Note that the distance between two disjoint simplices in $\Delta \subset \ell_{2}$ of dimension $\leq n$ is at least $\sqrt{\frac{2}{n+1}}$. For every chain $0 \leq l \leq k \leq k^{\prime}$ we denote by $D_{l, k, k^{\prime}}$ the union of standard simplices $\Delta^{k} \cup \Delta^{k^{\prime}}$ in $\ell_{2}$ with the intersection $\Delta^{k} \cap \Delta^{k^{\prime}}=\Delta^{l}$. Let $c_{l, k, k^{\prime}}$ be the Lipschitz constant of the identity map id $:\left(D_{l, k, k^{\prime}}, d_{\ell_{2}}\right) \rightarrow\left(D_{l, k, k^{\prime}}, d_{\text {geod }}\right)$ where $d_{\text {geod }}$ is the intrinsic metric on $D_{l, k, k^{\prime}}$ induced by the Euclidean metric. Let $\bar{c}=\max \left\{c_{l, k, k^{\prime}} \mid k^{\prime} \leq 2 n+1\right\}$. We take $c=b \sqrt{n+1} \bar{c}$. Then for every couple of points $x, x^{\prime} \in K$ with $\left\|x-x^{\prime}\right\| \geq \sqrt{\frac{2}{n+1}}$ we obtain $d_{L}\left(f(x), f\left(x^{\prime}\right)\right) \leq b \leq c\left\|x-x^{\prime}\right\| \leq c \lambda\left\|x-x^{\prime}\right\|$. Assume that $x \in \Delta^{k} \subset K, x^{\prime} \in \Delta^{k^{\prime}} \subset K$, and $\left\|x-x^{\prime}\right\|<\sqrt{\frac{2}{n+1}}$ where $\Delta^{k}, \Delta^{k^{\prime}}$ are simplices. Then $\Delta^{k} \cap \Delta^{k^{\prime}} \neq \emptyset$. Hence there is a geodesic segment $J$ in $\Delta^{k} \cup \Delta^{k^{\prime}}$ joining $x$ with $x^{\prime}$ of length $|J|$. We may assume that it is piece-wise linear (actually it consists of two straight intervals). Since $f$ is $\lambda$-Lipschitz on each of the segments of $J$, we obtain $d_{L}\left(f(x), f\left(x^{\prime}\right)\right) \leq \lambda|J| \leq \lambda c\left\|x-x^{\prime}\right\|$.

Let $f: X \rightarrow \Delta$ be a map of a metric space to the standard simplex. Denote by

$$
\delta(f)=\inf \left\{\operatorname{Lip}(\psi)|\psi: X \rightarrow \partial \Delta, \psi|_{f^{-1}(\partial \Delta)}=\left.f\right|_{f^{-1}(\partial \Delta)}\right\}
$$

Let $X$ be a proper geodesic metric space with an anti-Čech approximation $\left\{p_{i}: X \rightarrow\right.$ $\left.N_{i}\right\}$ where $\operatorname{dim} N_{i}=n$ for all $i$. For every $i$ we define

$$
\delta^{i}=\varlimsup_{\Delta \rightarrow \infty} \delta\left(\left.p_{i}\right|_{p_{i}^{-1}(\Delta)}\right)
$$

where $\Delta$ runs over all $n$-simplices from $N_{i}$.

Proposition 3.2. Suppose that $\operatorname{asdim} X=n$ for a proper geodesic metric space $X$ and let $\left\{p_{i}: X \rightarrow N_{i}\right\}$ to be an anti-Čech approximation of $X$ by $n$-dimensional polyhedra. Then there is $c>0$ such that $\delta^{i}>c$ for all $i$.

Proof. Assume the contrary. By passing to a subsequence we may assume that $\delta^{i} \rightarrow 0$. Let $C_{i} \subset N_{i}$ be a finite subcomplex such that $\delta\left(\left.p_{i}\right|_{p_{i}^{-1}(\Delta)}\right)<2 \delta^{i}$ for all $n$-simplices $\Delta \subset N_{i} \backslash C_{i}$. Then by sweeping one can define a map $\xi_{i}: X \rightarrow N_{i}^{(n-1)} \cup C_{i}$ which is $2 \delta^{i}$ Lipschitz on every set of the from $p_{i}^{-1}(\Delta)$. Since $X$ is geodesic, the map $\xi_{i}$ is $2 \delta^{i}$-Lipschitz where $N_{i}^{(n-1)} \cup C_{i}$ is taken with restricted metric from $N_{i}$ and $N_{i}$ is supplied with the uniform geodesic metric. Consider the quotient map $q_{i}: N_{i}^{(n-1)} \cup C_{i} \rightarrow N_{i}^{(n-1)} \cup C_{i} / C_{i}=$ $K_{i}$ and take a uniform bounded metric on $K_{i} \subset \ell_{2}\left(K^{(0)}\right)$. By Proposition $3.1 q_{i}$ is $c^{-}$ Lipschitz where $c$ depends on $n$ only. Thus, $\psi_{i}=q_{i} \circ \xi_{i}: X \rightarrow K_{i}$ is $2 c \delta^{i}$-Lipschitz. 
Therefore, $\left\{\psi_{i}: X \rightarrow K_{i}\right\}$ is an anti-Čech approximation $\left\{\psi_{i}: X \rightarrow K_{i}\right\}$ of $X$ by $n-1$ dimensional polyhedra. Then by the definition $\operatorname{asdim} X \leq n-1$ (see the discussion after Lemma 2.1), which contradicts to the assumption of the Proposition.

The following lemma is taken from [Dr4], Lemma 2.2.

Lemma 3.3. Suppose that $X$ and $Y$ are finite uniform simplicial complexes. Then for every $\lambda$ there exists $\mu=\mu(\lambda)$ such that every null homotopic $\lambda$-Lipschitz map $f: X \rightarrow Y$ admits a $\mu$-Lipschitz homotopy $H: X \times I \rightarrow Y$ to a constant map.

Here we give a short review of the classical extension theory. Let $(K, A)$ be a $\mathrm{CW}$ complex pair. Suppose that an extension problem

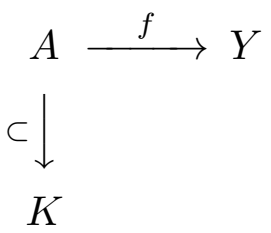

for a simply connected space $Y$ is resolved on the $n$-skeleton $K^{(n)}$ of $K$ by a map $g$ : $A \cup K^{(n)} \rightarrow Y$. The map $g$ defines the obstruction cochain $c_{g}: C_{n+1}(K, A) \rightarrow \pi_{n}(Y)$ which is a relative cocycle. If a corresponding relative cohomology class is zero, i.e., if $c_{g}=\delta \gamma$ for some $\gamma$, then one can change the map $g$ on the relative $n$-skeleton without changing it on the $n$-1-dimensional skeleton such that a new map $g^{\prime}$ can be extended to a map $\bar{g}: A \cup K^{(n+1)} \rightarrow Y[\mathrm{Hu}]$.

If the homotopy group $\pi_{n}(Y)$ is supplied with a norm and $Y$ is given some metric one can bring a quantitative statement.

Proposition 3.4. Suppose that $K$ is a simplicial complex supplied with a uniform metric and let $Y=S^{n}$ be the unit $n$-sphere. Suppose that in the above extension problem $g$ is $\lambda$-Lipschitz and $|\gamma| \leq b$. Then $g^{\prime}$ can be taken $\mu^{\prime}$-Lipschitz and $\bar{g}$ with a $\mu$-Lipschitz restriction to every $n+1$-simplex where $\mu^{\prime}, \bar{\mu}$ depend on $n, \lambda$ and $b$ only.

Proof. We fix a triangulation on $S^{n}$, say, by identifying $S^{n}$ with the boundary of the standard $n+1$-simplex. Let $\sigma \subset S^{n}$ be an $n$-face. Using a simplicial approximation we may assume that $g$ is simplicial with respect to some iterated barycentric subdivision $\beta^{i}\left(A \cup K^{(n)}\right)$ where $i$ depends on $\lambda$ (and $n$ ). The Obstruction Theory [Hu] prescribes a construction of $g^{\prime}$ as a map having degree on each $n$-simplex $\sigma^{\prime}$ in $K \backslash A$ equal to $\operatorname{deg}\left(\left.g\right|_{\sigma^{\prime}}\right)-\gamma\left(\sigma^{\prime}\right)$ where the degree of $\left.g\right|_{\sigma^{\prime}}$ ) (as well as the degree of $\left.g^{\prime}\right|_{\sigma^{\prime}}$ ) is computed for the map of pairs $\left.g\right|_{\sigma^{\prime}}:\left(\sigma^{\prime}, \partial \sigma^{\prime}\right) \rightarrow\left(S^{n}, S^{n} \backslash I n t \sigma\right)$. Since $\left|\operatorname{deg}\left(\left.g\right|_{\sigma^{\prime}}\right)\right|$ is bounded by the number of simplices in $\beta^{i}\left(\Delta^{n}\right)$. Thus, $\left|\operatorname{deg}\left(\left.g\right|_{\sigma^{\prime}}\right)-\beta\left(\sigma^{\prime}\right)\right|$ is uniformly bounded by 
a number that depends only on $n, \lambda$ and $b$. Since every $\sigma^{\prime}$ is isometric to the standard $n$-simplex, this degree can be realized by a $\mu^{\prime}$-Lipschitz map $g^{\prime}$ where $\mu^{\prime}$ depends on $n, \lambda$ and $b$ only. According to Lemma 3.3 the extension $\bar{g}$ of $g^{\prime}$ can be taken to be $\bar{\mu}$-Lipschitz on every $n+1$-dimensional simplex of $K$.

Let $\sqcup \Delta_{i}^{n}$ be a disjoint union of (oriented) $n$-simplices. Then the $n$-cochain $\mu$ that take each simplex to 1 defines a nonzero element $\mu \in A H_{b}^{n}\left(\sqcup \Delta_{i}^{n}, \sqcup \partial \Delta_{i}^{n}\right)$ which we call the fundamental class.

The family $\left\{A_{i}\right\}$ of bounded subsets in a metric space $X$ is called dispersed if there is a function $s: \mathbf{R}_{+} \rightarrow \mathbf{R}_{+}$tending to infinity such that the family $\left\{A_{i} \backslash B_{R}\left(x_{0}\right)\right\}$ is $s(R)$-disjoint.

Lemma 3.5. Suppose that $\operatorname{asdim} X=n$ for a proper geodesic metric space $X$ and let $\left\{p_{i}: X \rightarrow N_{i} ; p_{i+1}^{i}, q_{i+1}^{i}\right\}$ be a regular anti-Čech approximation of $X$ by $n$-dimensional simplicial complexes. Then there is a sequence of $n$-simplices $\Delta_{i} \subset N_{i}$ such that the cohomology homomorphism induced by $\phi=\left.\sqcup p_{i}\right|_{p_{i}^{-1}\left(\Delta_{i}\right)}$,

$$
\phi^{*}: A H_{b}^{n}\left(\sqcup \Delta_{i}, \sqcup \partial \Delta_{i}\right) \rightarrow A H^{n}\left(\sqcup p_{i}^{-1}\left(\Delta_{i}\right), \sqcup p_{i}^{-1}\left(\partial \Delta_{i}\right)\right),
$$

takes the fundamental class $\mu$ to a nonzero element.

Proof. In view of Proposition 3.2 we can take $\Delta_{i} \subset N_{i}$ such that $\delta\left(\left.p_{i}\right|_{p_{i}^{-1}\left(\Delta_{i}\right)}\right)>c / 2$ and $\left\{p_{i}^{-1}\left(\Delta_{i}\right)\right\}$ is dispersed.

Denote by $M_{i}^{k}=\left(p_{i}^{k}\right)^{-1}\left(\Delta_{i}\right)$, and $K_{i}^{k}=\left(p_{i}^{k}\right)^{-1}\left(\partial \Delta_{i}\right), k<i$. According to the condition (2) of a regular anti-Cech approximation $M_{i}^{k}$ is a subcomplex of $N_{k}$. For every function $\kappa: \mathbf{N} \rightarrow \mathbf{N}$ with $\kappa=\kappa(i)<i$ and $\kappa(i) \rightarrow \infty$ we define an open cover $\mathcal{U}_{\kappa}$ of $\sqcup p_{i}^{-1}\left(\Delta_{i}\right)$ as follows

$$
\mathcal{U}_{\kappa}=\left\{p_{\kappa(i)}^{-1}\left(O s t\left(v, M_{i}^{\kappa(i)}\right)\right) \mid v \in\left(M_{i}^{\kappa(i)}\right)^{(0)}, i \in \mathbf{N}\right\}
$$

where $\operatorname{Ost}(v, M)$ denotes the open star of a vertex $v$ in a complex $M$. Let $\left\{\mathcal{U}_{i}\right\}$ be the family of open covers that forms the above anti-Čech approximation. It is easy to check that for any $j$ and for every subcomplex $L \subset N_{j}, p_{j}^{-1}\left(O s t(u, L)=U \cap p_{j}^{-1}(L)\right.$ where $U \in \mathcal{U}_{j}$ and $u$ a vertex in the nerve $N_{j}$ that corresponds to $U$. Then $\mathcal{U}_{\kappa}=$ $\left.\cup_{i} \mathcal{U}_{\kappa(i)}\right|_{p_{i}^{-1}\left(\Delta_{i}\right)}$. Then the nerve of $\mathcal{U}_{\kappa}$ coincides with $\sqcup_{i} M_{i}^{\kappa(i)}$. Being the composition of simplicial approximations the map $q_{i}^{k}$ is a simplicial approximation of $p_{i}^{k}$. Thus, we obtain $q_{i}^{k}\left(M_{i}^{k}\right)=\Delta_{i}$ for all $k<i$. 
Since $\left\{p_{i}^{-1}\left(\Delta_{i}\right)\right\}$ is dispersed, for every $\mathcal{U} \in a \operatorname{Cov}\left(\sqcup p_{i}^{-1}\left(\Delta_{i}\right)\right)$ we obtain that the Lebesgue number of the restrictions tends to infinity: $L\left(\left.\mathcal{U}\right|_{p_{i}^{-1}\left(\Delta_{i}\right)}\right) \rightarrow \infty$. From here it is easy to verify that the family of covers

$$
\left\{\mathcal{U}_{\kappa} \mid \kappa: \mathbf{N} \rightarrow \mathbf{N}, \kappa=\kappa(i)<i, \kappa(i) \rightarrow \infty\right\}
$$

is cofinal in $a \operatorname{Cov}\left(\sqcup p_{i}^{-1}\left(\Delta_{i}\right)\right)$. Note that $N\left(\left.\mathcal{U}_{\kappa(i)}\right|_{p_{i}^{-1}\left(\partial \Delta_{i}\right)}\right)=K_{i}^{\kappa(i)}$.

Assume that $\phi^{*}(\mu)=0$. Then there is $\kappa: \mathbf{N} \rightarrow \mathbf{N}, \kappa=\kappa(i)<i$ and $\kappa(i) \rightarrow \infty$ such that the homomorphism $\psi^{*}$ induced by the simplicial map

$$
\psi=\left.\sqcup q_{i}^{\kappa(i)}\right|_{M_{i}^{\kappa(i)}}: \sqcup\left(M_{i}^{\kappa(i)}, K_{i}^{\kappa(i)}\right) \rightarrow \sqcup\left(\Delta_{i}, \partial \Delta_{i}\right)
$$

takes $\mu$ to zero. Let $\psi_{i}=\left.q_{i}^{\kappa(i)}\right|_{M_{i}^{\kappa(i)}}$. Denote by $\mu_{i}$ the image of the cocycle $1_{i} \in$ $C^{n}\left(\Delta_{i}, \partial \Delta_{i}\right) \cong \mathbf{Z}$ that takes $\Delta_{i}$ to 1 under $\psi_{i}^{*}$. If $\phi_{1}^{*}(\mu)=0$ then for all sufficiently large $i$ there are a number $b>0$ and $b$-bounded $n$-1-dimensional cochains $\gamma_{i} \in C^{n-1}\left(M_{i}^{\kappa(i)}\right)$ such that $\delta \gamma_{i}=\mu_{i}$. Since $q_{i}^{k(i)}$ is a simplicial approximation of $p_{i}^{\kappa(i)}$, we obtain that $\psi_{i}\left(K_{i}^{\kappa(i)}\right) \subset \partial \Delta_{i}$. We consider the extension problem:

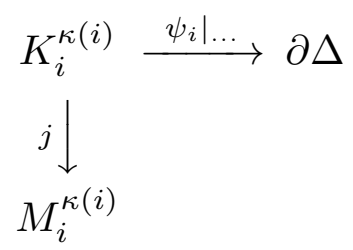

where $j: K_{i}^{\kappa(i)} \rightarrow M_{i}^{\kappa(i)}$ is the inclusion. The map $\psi_{i}$ solves this problem on the $n-1$ skeleton and the relative cocycle $\mu_{i}$ is the obstruction cocycle to the solution of the problem on $M_{i}^{\kappa(i)}$. Since the obstruction cocycle is a coboundary, by Proposition 3.4 one can change the map $\psi_{i}$ on the $(n-1)$-skeleton of $M_{i}^{\kappa(i)} \backslash K_{i}^{\kappa(i)}$ without changing it on the $(n-2)$-skeleton such that new map has an extension to $M_{i}^{\kappa(i)}$. Moreover, we may assume that there is $\bar{\lambda}$ such that for every $i$ there is an extension $\bar{\psi}_{i}: M_{i}^{\kappa(i)} \rightarrow \partial \Delta_{i}$ which is $\bar{\lambda}$-Lipschitz on every $n$-simplex of $M_{i}^{\kappa(i)}$. By Proposition 3.1 we can conclude that $\bar{\psi}_{i}$ is $c^{\prime} \bar{\lambda}$-Lipschitz on $M_{i}^{\kappa(i)}$ for all $i$. Note that

$$
c / 2<\delta\left(\left.\partial_{i}\right|_{p_{i}^{-1}\left(\Delta_{i}\right)}\right) \leq \operatorname{Lip}\left(\bar{\psi}_{i} \circ p_{\kappa(i)}\right)<c^{\prime} \bar{\lambda} \epsilon_{\kappa(i)} .
$$

This contradict to the fact that $\epsilon_{\kappa(i)} \rightarrow 0$.

Asymptotic Alexandroff Theorem. The classical Alexandroff Theorem states that $\operatorname{dim} X=\operatorname{dim}_{\mathbf{Z}} X$ for compact metric spaces provided $\operatorname{dim} X<\infty$. Here we prove the following. 
Theorem 3.6. Suppose $\operatorname{asdim} X<\infty$ for a proper geodesic metric space $X$. Then $\operatorname{asdim}_{\mathbf{Z}} X=\operatorname{asdim} X$.

Proof. The inequality $\operatorname{asdim}_{\mathbf{Z}} X \leq \operatorname{asdim} X$ follows from Proposition 2.1 and the definition of $\operatorname{asdim}_{\mathbf{Z}} X$.

Assume that $\operatorname{asdim} X=n$. Then by Lemma $3.5 A H^{n}(Z, Y) \neq 0$ for some $Y \subset Z \subset X$. Therefore, $\operatorname{asdim}_{\mathbf{Z}} X \geq n$.

Lemma 3.7. Suppose asdim $X<\infty$ for a uniformly path connected metric space $X$ and let $\operatorname{asdim}_{G} X=n$ for some abelian group $G$. Then there exists a dispersed family $\left\{U_{i}\right\}$ of bounded open sets in $X$ such that $A H^{n}\left(\sqcup \bar{U}_{i}, \sqcup \partial U_{i} ; G\right) \neq 0$.

Proof. Let asdim $X=n$ and let $W \subset X$ be an open subset such that $A H^{n}(X, X \backslash W ; G) \neq$ 0 . There are dispersed families of bounded open sets $\mathcal{V}^{k}=\left\{U_{i}^{k}\right\}, k=0, \ldots, n$, such that the union $\mathcal{V}=\cup_{k=0}^{n} \mathcal{V}^{k}$ is a cover $\mathcal{V} \in a \operatorname{Cov}(X)$ (see [DKU] for the construction). Denote by $\mathcal{U}^{k}=\left.\mathcal{V}^{k}\right|_{W}$. Using induction and Propositions 2.4 and 2.5 we can derive that $A H^{n}\left(X, X \backslash \cup_{U \in \mathcal{U}^{k}} U ; G\right) \neq 0$ for some $k$. We take $\mathcal{U}^{k}$ as the desired family $\left\{U_{i}\right\}$. The excision (Proposition 2.6) implies that $A H^{n}\left(\sqcup \bar{U}_{i}, \sqcup \partial U_{i} ; G\right) \neq 0$.

\section{$\S 4$ Relation to the COARSE COHOMOlogical Dimension}

In this section we consider only metric spaces $Y$ that admit an anti-Čech approximation. Using an anti-Čech approximation $\left\{N_{i}, q_{i+1}^{i}\right\}$ of $Y$ John Roe [Ro1] defined coarse cohomology group $H X^{*}(Y)$ of $Y$ as the homology of the inverse limit of the cochain complexes $C_{0}^{*}\left(N_{i}\right)$ that consist of cochains with compact supports. Then the standard argument shows that coarse cohomology groups fit in the short exact sequence [Ro1]:

$$
0 \rightarrow \lim ^{1} H_{c}^{k-1}\left(N_{i}\right) \rightarrow H X^{k}(Y) \rightarrow \lim _{\leftarrow} H_{c}^{k}\left(N_{i}\right) \rightarrow 0
$$

where $H_{c}$ stands for the cohomology with compact supports. Let $A \subset Y$, denote by $A_{i}=S t\left(p_{i}(A), N_{i}\right)$. We may assume that $q_{i+1}^{i}\left(A_{i}\right) \subset A_{i+1}$. Then for relative coarse cohomology there is an exact sequence:

$$
(*) \quad 0 \rightarrow \lim ^{1} H_{c}^{k-1}\left(N_{i} \backslash A_{i}\right) \rightarrow H X^{k}(Y, A) \rightarrow \lim _{\leftarrow} H_{c}^{k}\left(N_{i} \backslash A_{i}\right) \rightarrow 0 .
$$

The coarse cohomology and this exact sequence are defined for any coefficient group. Using coarse cohomology with integral coefficients one can define a coarse cohomological dimension [Dr1]

$$
\mathrm{X}-\operatorname{dim} Y=\max \left\{k \mid H X^{k}(Y, A) \neq 0 \text { for some } A \subset Y\right\}-1 \text {. }
$$


The shift by one is needed to get the equality $X-\operatorname{dim} \mathbf{R}^{n}=n$. We note that in [Dr1] this dimension was defined under a different name. Also in [Dr1] it was suggested to make a shift in the grading of the coarse cohomology in order to achieve the equality $X-\operatorname{dim} \mathbf{R}^{n}=n$. Here we embed this shift in the definition of $X-\operatorname{dim}$.

Proposition 4.1. Let $Y$ be a proper metric space, then

$$
\mathrm{X}-\operatorname{dim} Y \leq \operatorname{asdim} Y
$$

Proof. Assume that asdim $Y \leq n$. There is an anti-Čech approximation of $Y$ with polyhedra $N_{i}$ of dimension $\leq n$. Then $H_{c}^{k}\left(N_{i}, A_{i}\right)=0$ for any $A_{i}$ for $k>n$. Therefore, $H X^{k}(Y, A)=0$ for $k>n+1$. Hence $X-\operatorname{dim} Y \leq n$.

Proposition 4.2. For every metric space $Y$,

$$
\mathrm{X}-\operatorname{dim}(Y \times \mathbf{R})=\mathrm{X}-\operatorname{dim} Y+1 .
$$

Proof. Let $\mathrm{X}-\operatorname{dim} Y=n$ and let $H X^{n}(Y, A) \neq 0$. Since for all $k$,

$$
H_{c}^{k}\left(N_{i} \backslash A_{i}\right)=H_{c}^{k+1}\left(\left(N_{i} \backslash A_{i}\right) \times \mathbf{R}\right),
$$

we obtain that $H X^{n+1}(Y \times R, A \times \mathbf{R}) \neq 0$. Thus, $\mathrm{X}-\operatorname{dim}(Y \times \mathbf{R}) \geq \mathrm{X}$-dim $Y+1$. In view of the inequality $\operatorname{asdim}(Y \times \mathbf{R}) \leq \mathrm{X}-\operatorname{dim} Y+1$, Proposition 4.1 implies the inequality $\mathrm{X}-\operatorname{dim}(Y \times \mathbf{R}) \leq \mathrm{X}-\operatorname{dim} Y+1$.

We note that the coarse cohomological dimension can be defined with any (abelian) coefficient groups $G$. We will use the notation $\mathrm{X}-\operatorname{dim}_{G} Y$.

Let $\left\{G_{i}, \phi_{i}^{i+1}\right\}$ be an inverse system with bonding maps $\phi_{i}^{i+1}: G_{i+1} \rightarrow G_{i}$. For $k>i$ we denote by $\phi_{i}^{k}: G_{k} \rightarrow G_{i}$ the composition $\phi_{i}^{i+1} \circ \cdots \circ \phi_{k-1}^{k}$ and denote by

$$
\phi_{i}^{\infty}: \lim _{\leftarrow} G_{j} \rightarrow G_{i}
$$

the projection from the limit space to the $i$ th factor.

Proposition 4.3. Suppose that for an inverse sequence of countable groups

$$
\lim _{\leftarrow}^{1}\left\{G_{i}, \phi_{i}^{i+1}\right\} \neq 0 .
$$


Then there $i s i_{0}$ such that for every $i \geq i_{0}$ there is an element $\alpha_{i} \in G_{i}$ with $\phi_{i_{0}}^{i}\left(\alpha_{i}\right) \neq 0$ and $\alpha_{i} \notin \operatorname{Im}\left(\phi_{i}^{\infty}\right)$.

Proof. Since $\lim ^{1} G_{i}$ is nonzero, the Mittag-Lefler condition does not hold for the system. Therefore there is $i_{0}$ such that the nested sequence

$$
\operatorname{Im}\left(\phi_{i_{0}}^{i_{0}+1}\right) \supset \operatorname{Im}\left(\phi_{i_{0}}^{i_{0}+2}\right) \supset \operatorname{Im}\left(\phi_{i_{0}}^{i_{0}+3}\right) \supset \ldots
$$

does not stabilize. In view of this we can take $\alpha_{i}$ such that $\phi_{i_{0}}^{i}\left(\alpha_{i}\right) \in \operatorname{Im}\left(\phi_{i_{0}}^{i}\right) \backslash \operatorname{Im}\left(\phi_{i_{0}}^{k}\right)$ for some $k$.

Proposition 4.4. Suppose that a geodesic metric space $Y$ that admits an anti-Čech approximation $\left\{N_{i}\right\}$ satisfies the equality $X-\operatorname{dim}_{G} Y=n$ and let $H^{n+1}(Y, A ; G) \neq 0$ for some $A$ and some countable group $G$. Then the homomorphism

$$
\lim _{\leftarrow}^{1} H_{c}^{n}\left(N_{i} \backslash A_{i} ; G\right) \stackrel{\cong}{\longrightarrow} H X^{n+1}(X, A ; G)
$$

in the short exact sequence $\left(^{*}\right)$ is an isomorphism.

Proof. We assume that all $N_{i}$ are given the uniform geodesic metric.

Assume the contrary. Let $\alpha \in \lim _{\leftarrow} H_{c}^{n+1}\left(N_{i} \backslash A_{i} ; G\right)$ and $\alpha \neq 0$. Let $\left(\alpha_{i}\right)$ be a thread representing $\alpha, \alpha_{i} \in H_{c}^{n+1}\left(N_{i} \backslash A_{i} ; G\right)$. From the definition of cohomology with compact supports it follows that there is a bounded open set $U_{1} \subset N_{1} \backslash A_{1}$ and an element $\gamma_{1} \in H_{c}^{n+1}\left(U_{1} ; G\right)$ which is taken by the inclusion homomorphism to $\alpha_{1}$. There is $r_{1}>0$ such that $U_{1}$ lies in the $r_{1} / 2$-neighborhood of $A_{1}, U_{1} \subset N_{r_{1} / 2}\left(A_{1}\right)$. Let $W_{1}=p_{1}^{-1}\left(U_{1}\right)$.

Since the projection $p_{1}: X \rightarrow N_{1}$ is uniformly cobounded, the set $A^{1}=p_{1}^{-1}\left(N_{r_{1}}\left(A_{1}\right)\right)$ is in bounded distance to $A$. Then $H X^{n+1}\left(Y, A^{1} ; G\right) \cong H X^{n+1}(Y, A ; G)$. Moreover for large enough $k$ there is an isomorphism of inverse sequences

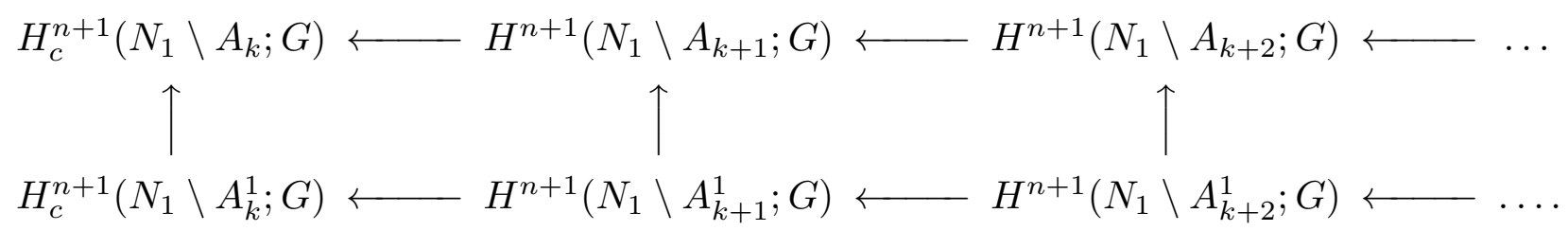

There is a bounded open set $U_{2} \subset N_{k} \backslash A_{k}^{1}$ and an element $\gamma_{2} \in H_{c}^{n+1}\left(U_{2} ; G\right)$ that goes to $\alpha_{k}$ under the inclusion homomorphism. Let $U_{1}^{2}=\left(q_{1}^{2}\right)^{-1}\left(U_{2}\right)$ and $W_{2}=p_{1}^{-1}\left(U_{2}^{1}\right)$. The commutative diagram

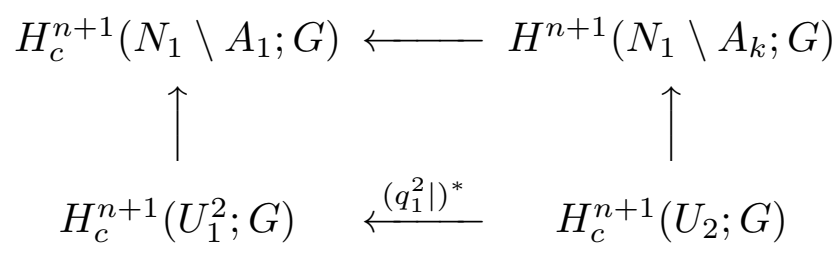


implies that $\left(q_{1}^{2} \mid\right)^{*}\left(\gamma_{2}\right) \neq 0$. There is $r_{2}>0$ such that $U_{2}$ lies in $r_{2} / 2$-neighborhood of $A_{k}$ and we continue in a similar fashion. As the result we construct a dispersed sequence of bounded open sets $W_{i} \subset Y$ and an anti-Čech approximation

$$
\left(N_{1}, N_{1} \backslash\left(\sqcup_{j} U_{1}^{j}\right)\right) \rightarrow\left(N_{k_{2}}, N_{k_{2}} \backslash\left(\sqcup_{j} U_{2}^{j}\right)\right) \rightarrow\left(N_{k_{3}}, N_{k_{3}} \backslash\left(\sqcup_{j} U_{3}^{j}\right)\right) \rightarrow \ldots
$$

of the pair $\left(Y, Y \backslash\left(\sqcup_{i} W_{i}\right)\right)$ such that for all $i$ there are nontrivial elements $\gamma_{i} \in H_{c}^{n+1}\left(U_{i} ; G\right)$ that survive after translation to the first level. We may assume that these elements do not belong to the image of the projection from the higher level. This implies that the system is not Mittag-Lefler and hence $\lim ^{1} H_{c}^{n+1}\left(U_{i} ; G\right) \neq 0$. Therefore, $H X^{n+2}(Y, Y \backslash$ $\left.\left(\sqcup W_{i}\right) ; G\right) \neq 0$ and hence $\mathrm{X}-\operatorname{dim}_{G} Y \geq n+1$. This contradicts to the assumption.

A metric space $Y$ is uniformly $n$-connected if there is a control function $\rho: \mathbf{R}_{+} \rightarrow \mathbf{R}_{+}$, $\rho(t) \geq t$, such that for every $t>0$ and every $y \in Y$ the inclusion $B_{t}(y) \rightarrow B_{\rho(t)}(y)$ induces zero homomorphism of $k$-dimensional homotopy groups for $k \leq n$. It is called uniformly contractible if there is a function $\rho: \mathbf{R}_{+} \rightarrow \mathbf{R}_{+}, \rho(t) \geq t$, such that for every $t>0$ and every $y \in Y$ the inclusion $B_{t}(y) \rightarrow B_{\rho(t)}(y)$ is null-homotopic.

We recall that for every $Y$ there is a natural through homomorphism $c: H X^{k}(Y ; G) \rightarrow$ $H_{c}^{k}(Y ; G)[\mathrm{Ro} 1]$ :

$$
H X^{k}(Y ; G) \rightarrow \lim _{\leftarrow} H_{c}^{k}\left(N_{i} ; G\right) \rightarrow H_{c}^{k}(Y ; G) .
$$

Theorem 4.5 ([Ro1], page 33). For a uniformly n-connected metric space $Y$ the map $c: H X^{k}(Y ; G) \rightarrow H_{c}^{k}(Y ; G)$ is an isomorphism for $k \leq n$ and for any abelian coefficients group $G$.

We denote the global cohomological dimension of a space $Y$ with respect to the coefficient group $G$ by

$$
\operatorname{gcd}_{G} Y=\max \left\{n \mid H_{c}^{n}(Y ; G) \neq 0\right\} .
$$

Proposition 4.6. Let $Y$ be a uniformly n-connected metric space of bounded geometry and let gld $_{G} Y \leq n$ for a countable group $G$. Then $\mathrm{X}-\operatorname{dim}_{G} Y \geq \operatorname{gld}_{G} Y$.

Proof. Assume the contrary: $\mathrm{X}-\operatorname{dim}_{G} Y=m<k=\operatorname{gld}_{G} Y$. In view of Theorem 4.5 $H X^{k}(Y ; G)=H_{c}^{k}(Y ; G) \neq 0$ and hence, $m \geq k-1$. Then $m=k-1$. Since $Y$ has bounded geometry, it has an anti-Čech approximation $\left\{N_{i}\right\}$. By Proposition 4.4 and Theorem 4.5 we obtain

$$
\lim _{\leftarrow}^{1} H_{c}^{k}\left(N_{i} ; G\right)=H X^{k}(Y ; G)=H_{c}^{k}(Y ; G) .
$$

The last group is countable as the direct limit of countable groups. The first group is uncountable as a nonzero group which is a $\lim ^{1}$ group of a sequence of countable groups [Ha]. We arrived to a contradiction. 
Proposition 4.7. Suppose that $\mathrm{X}-\operatorname{dim}_{G} Y=n$ for a proper geodesic metric space $Y$ and a countable group $G$. Then there is a dispersed sequence $\left\{U_{i}\right\}$ of bounded open sets in $Y$ such that $H X^{n+1}\left(\sqcup \bar{U}_{i}, \sqcup \partial U_{i} ; G\right) \neq 0$. Moreover, there is an anti-ČCh approximation $\left\{N_{i}, p_{i}\right\}$ of $Y$ such that $U_{i}=p_{i}^{-1}\left(V_{i}\right), V_{i}=\operatorname{Int}\left(L_{i}\right)$, and $L_{i} \subset N_{i}$ is a finite subcomplex.

Proof. Assume that $H X^{n+1}(Y, A ; G) \neq 0$. Let

$$
N_{1} \stackrel{q_{2}^{1}}{\longrightarrow} N_{2} \stackrel{q_{3}^{2}}{\longrightarrow} N_{3} \stackrel{q_{4}^{3}}{\longrightarrow} \ldots N_{i} \stackrel{q_{i+1}^{i}}{\longrightarrow} \ldots
$$

be a regular anti-Čech approximation of $Y$. By Proposition 4.4,

$$
\lim _{\leftarrow}^{1} H^{n}\left(N_{i}, A_{i} ; G\right) \neq 0 .
$$

Note that $q_{i+1}^{i}:\left(N_{i}, A_{i}\right) \rightarrow\left(N_{i+1}, A_{i+1}\right)$ is homotopic to $p_{i+1}^{i}:\left(N_{i}, A_{i}\right) \rightarrow\left(N_{i+1}, A_{i+1}\right)$. Thus, $\left(q_{i+1}^{i}\right)^{*}=\left(p_{i+1}^{i}\right)^{*}$.

We apply Proposition 4.3 to obtain $i_{0}$ and $\alpha_{i} \in H^{n}\left(N_{i}, A_{i} ; G\right)$. Without loss of generality we may assume that $i_{0}=1$. Then $\left(p_{i}^{1}\right)^{*}\left(\alpha_{i}\right) \neq 0$ and $\alpha_{i} \notin \operatorname{Im}\left(p_{\infty}^{i}\right)^{*}$ where

$$
\left(p_{\infty}^{i}\right)^{*}: \lim _{\leftarrow} H^{n}\left(N_{k}, A_{k} ; G\right) \rightarrow H^{n}\left(N_{i}, A_{i} ; G\right)
$$

is the projection from the limit to $i$ th factor in the inverse sequence

$$
\left\{H^{n}\left(N_{k}, A_{k} ; G\right),\left(p_{k+1}^{k}\right)^{*}\right\} .
$$

Since $H^{n}\left(N_{k}, A_{k} ; G\right)=H_{c}^{n}\left(N_{k} \backslash A_{k} ; G\right)$ there is a open set $\left.V_{k} \subset N_{k} \backslash A_{k}\right)$ with compact closure $\bar{V}_{k}$ and an element $\beta_{k} \in H_{c}^{n}\left(V_{k} ; G\right)$ such that $\beta_{k}$ goes to $\alpha_{k}$ under the inclusion homomorphism. We may assume that $V=\operatorname{Int} L_{k}$ where $L_{k}$ is a finite subcomplex of $N_{k}$. We define $U_{i}=p_{i}^{-1}\left(V_{i}\right)$. For $j \geq i$ we denote by $L_{i}^{j}=\left(p_{j}^{i}\right)^{-1}\left(L_{j}\right)$. For $j<i$ we denote $L_{i}^{j}=N\left(\left.\mathcal{U}_{j}\right|_{\bar{U}_{j}}\right)$, the nerve of the cover generating $N_{j}$ restricted to $\bar{U}_{j}$. In view of $\left(^{*}\right)$ it suffices to show that $\lim ^{1} H^{n}\left(\sqcup_{j}\left(L_{i}^{j}, \partial L_{i}^{j}\right) ; G\right) \neq 0$. The inverse sequence $\left\{H^{n}\left(\sqcup_{j \geq 1}\left(L_{i}^{j}, \partial L_{i}^{j}\right) ; G\right)\right\}$ can be mapped epimorphically onto the sequence $\left\{H_{c}^{n}\left(\sqcup_{j \geq i}\left(L_{i}^{j}, \partial L_{i}^{j}\right) ; G\right)\right\}$. In view of the 6 -term exact sequence for the inverse limit it suffices to show that

$$
\lim _{\leftarrow}^{1}\left\{H_{c}^{n}\left(\sqcup_{j \geq i}\left(L_{i}^{j}, \partial L_{i}^{j}\right) ; G\right)\right\} \neq 0 .
$$

Since for $j>i$ the element $\beta_{j}$ goes to nonzero, the result follows.

Coarse cohomological dimension vs asymptotic. On subgroups of the reals $\mathbf{R}$ we consider a natural norm || . On the $\bmod p$ group $\mathbf{Z}_{p}$ we consider the zero semi-norm. 
Theorem 4.8. The following holds for every proper metric space $Y$ with finite asymptotic dimension:

(1) $\operatorname{asdim}_{G} Y \geq \mathrm{X}-\operatorname{dim}_{G} Y$ for every subgroup $G \subset \mathbf{Q}$;

(2) $\operatorname{asdim}_{\mathbf{Z}_{p}} Y=\mathrm{X}-\operatorname{dim}_{\mathbf{z}_{p}} Y$ for all $p$.

Proof. (1) If $G=\mathbf{Z}$, the result follows from Theorem 3.6 and Proposition 4.1.

Now we assume that $G$ is $p$-divisible for some $p$. Let $\mathrm{X}-\operatorname{dim}_{G} Y=n$ and let $U_{i}$ be as in Proposition 4.5. Let

$$
\sqcup_{i}\left(N_{1}^{i}, K_{1}^{i}\right) \stackrel{q_{2}^{1}}{\longrightarrow} \sqcup_{i}\left(N_{2}^{i}, K_{2}^{i}\right) \stackrel{q_{3}^{2}}{\longrightarrow} \sqcup_{i}\left(N_{3}^{i}, K_{3}^{i}\right) \rightarrow \ldots
$$

be an anti-Čech approximation of $\sqcup_{i}\left(\bar{U}_{i}, \partial U_{i}\right)$ where $N_{i}$ are finite complexes. Then $\lim ^{1} H_{c}^{n}\left(\sqcup_{i}\left(N_{j}^{i} \backslash K_{j}^{i}\right) ; G\right) \neq 0$. Note that each group $H_{c}^{n}\left(\sqcup_{i}\left(N_{j}^{i} \backslash K_{j}^{i}\right) ; G\right)=\oplus_{i} H^{n}\left(N_{j}^{i}, K_{j}^{i} ; G\right)$ is countable. Hence the system is not Mittag-Lefler. Without loss of generality, we may assume that for any $m$ there are $i \geq m$ and $k=k(i)$ and an element $\gamma_{i} \in$ $H^{n}\left(N_{k(i)}^{i}, K_{k(i)}^{i} ; G\right)$ such that $\left(q_{k(i)}^{1}\right)^{*}\left(\gamma_{i}\right) \neq 0$ and $\gamma_{i} \notin \operatorname{Im}\left(q_{k(i)+1}^{k(i)}\right)$. By dividing $\gamma_{i}$ by some power of $p$ we may achieve that $\gamma_{i}$ is represented by a cocycle with the norm $\leq 1$. This defines a nontrivial element of $A H_{b}^{n}\left(\sqcup_{i}\left(N_{k(i)}^{i}, K_{k(i)}^{i}\right) ; G\right)$ and of $A H^{n}\left(\sqcup \bar{U}_{i}, \sqcup \partial U_{i} ; G\right)$. Thus, $\operatorname{asdim}_{G} Y \geq n$.

(2) The same argument works to show that $\operatorname{asdim}_{\mathbf{Z}_{p}} Y \geq \mathrm{X}-\operatorname{dim}_{\mathbf{Z}_{p}} Y$.

Let $\operatorname{asdim}_{\mathbf{z}_{p}} Y=n$. We apply Lemma 3.7 to obtain a dispersed family of open set $\left\{U_{i}\right\}$ with $A H^{n}\left(\sqcup \bar{U}_{i}, \sqcup \partial U_{i} ; \mathbf{Z}_{p}\right) \neq 0$. Without loss of generality we may assume that $U_{i}=$ $p_{i}^{-1}\left(V_{i}\right)$ where $\left\{N_{i}, p_{i}\right\}$ is an anti-Čech approximation and $\bar{V}_{i} \subset N_{i}$ are subcomplexes. Furthermore, there is $i_{0}$ and

$$
\alpha \in H_{b}^{n}\left(\sqcup_{i \geq i_{0}}\left(\bar{V}_{i}, \partial V_{i}\right) ; \mathbf{Z}_{p}\right)=H^{n}\left(\sqcup_{i \geq i_{0}}\left(\bar{V}_{i}, \partial V_{i}\right) ; \mathbf{Z}_{p}\right)=\prod_{i \geq i_{0}} H^{n}\left(\bar{V}_{i}, \partial V_{i} ; \mathbf{Z}_{p}\right)
$$

such that $\alpha$ defines a nonzero element of $A H^{n}\left(\sqcup \bar{U}_{i}, \sqcup \partial U_{i} ; \mathbf{Z}_{p}\right) \neq 0$. If $\alpha=\left(\alpha_{i}\right)_{i \geq i_{0}}$, this implies that for every function $\kappa: \mathbf{N} \rightarrow \mathbf{N}, \kappa(i)>i$, the sequence $\left(\left(p_{i}^{\kappa(i)}\right)^{*}\left(\alpha_{i}\right)\right)_{i \geq i_{0}}$ is not eventually zero. We show that there is $k$ such that for $i>k$ the image $\left(p_{i}^{k}\right)^{*}\left(\alpha_{i}\right)$ is nonzero for infinitely many $i$. Let $J_{k}=\left\{i \in \mathbf{N} \mid\left(p_{i}^{k}\right)^{*}\left(\alpha_{i}\right) \neq 0\right\}$. Note that $J_{k} \subset$ $J_{k+1}$ and $i \in J_{i}$. If each $J_{k}$ is finite we can define $\kappa: \mathbf{N}_{\geq i_{0}} \rightarrow N_{\geq i_{0}}$ by the formula $\kappa\left(J_{k} \backslash J_{k-1}\right)=k-1$. Then $\kappa \rightarrow \infty$ and the sequence $\left(\left(p_{i}^{\kappa(i)}\right)^{*}\left(\alpha_{i}\right)\right)_{i \geq i_{0}}$ is zero which contradicts to the assumption.

Then it follows that $\lim _{\leftarrow}^{1} H_{c}^{n}\left(\cup_{j>i}\left(p_{i}^{j}\right)^{-1}\left(V_{i}\right) ; \mathbf{Z}_{p}\right) \neq 0$. Therefore $H X^{n+1}(Y, Y \backslash$ $\left.\cup U_{i} ; \mathbf{Z}_{p}\right) \neq 0$ and hence $\mathrm{X}-\operatorname{dim}_{\mathbf{z}_{p}} Y \geq n$. Thus, $\operatorname{asdim}_{\mathbf{z}_{p}} Y \leq \mathrm{X}-\operatorname{dim}_{\mathbf{Z}_{p}} Y$.

\section{Applications to dimension of discrete groups.}


Theorem 4.9. Let $\Gamma$ be a discrete group with finite $B \Gamma$, then $\mathrm{X}-\operatorname{dim} \Gamma=c d(\Gamma)$.

Proof. First we show that X-dim $\Gamma \geq \operatorname{cd}(\Gamma)$. Let $c d(\Gamma)=n$. Since $c d(\Gamma)=g l d_{\mathbf{Z}} \Gamma[\mathrm{Br}]$ and $E \Gamma$ is uniformly contractible, by Proposition 4.6 we obtain $\mathrm{X}-\operatorname{dim}_{\mathbf{Z}} E \Gamma=\mathrm{X}-\operatorname{dim}_{\mathbf{Z}} \Gamma \geq n$.

Let $\mathrm{X}-\operatorname{dim} \Gamma=n$ and let $c d \Gamma<n$. By crossing $\Gamma$ with $\mathbf{Z}$ and applying Proposition 4.2 we may assume that $n \geq 3$. Then we have $\operatorname{dim} E \Gamma<n$. Let $\left\{N_{i}, p_{i+1}^{i}, q_{i+1}^{i}\right\}$ be a regular anti-Cech approximation of $E \Gamma$. By Proposition 4.7 there is a dispersed sequence of open sets $U_{i}=p_{i}^{-1} V_{i}$ and elements $\alpha_{i} \in H_{c}^{n}\left(V_{i}\right)$ with $\left(p_{i}^{1}\right)^{*}\left(\alpha_{i}\right) \neq 0$. Let $W_{i}$ be the regular neighborhood of $\bar{V}_{i}$ in $N_{i}$, i.e., $W_{i}$ is the star neighborhood of $\bar{V}_{i}$ in the second barycentric subdivision of $N_{i}$. Let $A_{i}$ be the regular neighborhood of $\partial V_{i}$. Since the pair $\left(W_{i}, A_{i}\right)$ is homotopy equivalent to $\left(\bar{V}_{i}, \partial V_{i}\right)$, the element $\alpha_{i}$ lives in $H^{n}\left(W_{i}, A_{i}\right)$. Since $E \Gamma$ is uniformly contractible there are lifts $s_{i}: V_{i}^{1}=\left(p_{i}^{1}\right)^{-1}\left(\bar{V}_{i}\right) \rightarrow E \Gamma_{i}$ such that $p_{1} s_{i}$ is $r$-closed to the identity where $r$ is the same for all $i$. Since the Lipschitz constant of $p_{i}^{1}$ tends to zero, for large enough $i$ the maps $p_{i}^{1}:\left(\bar{V}_{i}^{1}, \partial V_{i}^{1}\right) \rightarrow\left(W_{i}, A_{i}\right)$ and $p_{i} s_{i}$ : $\left(\bar{V}_{i}^{1}, \partial V_{i}^{1}\right) \rightarrow\left(W_{i}, A_{i}\right)$ are homotopic. Hence $\left(p_{i}^{1}\right)^{*}\left(\alpha_{i}\right)=s_{i}^{*} p_{i}^{*}\left(\alpha_{i}\right)$. Since $\operatorname{dim} E \Gamma<n$, $s_{i}^{*}$ is zero homomorphism and hence $s_{i}^{*} p_{i}^{*}\left(\alpha_{i}\right)=0$. We arrived to a contradiction.

We recall that in the group theoretic language the groups with finite $B \Gamma$ are called the groups of the type $F L$. Also we recall that a finitely presented group is called of the type $\mathrm{FP}$ if $B \Gamma$ is dominated by a finite complex.

Proposition 4.10. Let $\Gamma$ be a discrete group of the type $F P$. Then $\operatorname{asdim} \Gamma \geq c d(\Gamma)$.

Proof. We may assume that $\operatorname{asdim} \Gamma<\infty$. Let $c d(\Gamma)=n$. Then $H^{n}(\Gamma ; \mathbf{Z} \Gamma) \neq 0$ $[\mathrm{Br}]$. The condition $\Gamma \in F P$ is equivalent to the existence of a homotopy domination $r: K \rightarrow B \Gamma$ by a finite complex. We may assume that $r$ induces an isomorphism of the fundamental groups and $\pi_{i}(K)=0$ for $1<i \leq n$. Then $H^{n}(\Gamma ; \mathbf{Z} \Gamma)=H_{c}^{n}(\tilde{K})$ where $\tilde{K}$ is the universal cover of $K$. Since the space $\tilde{K}$ is uniformly $n$-connected, by Proposition 4.6 we obtain $\mathrm{X}-\operatorname{dim}_{\mathbf{Z}} \tilde{K} \geq \operatorname{gld} \mathbf{Z} \tilde{K} \geq n$. Since $\tilde{K}$ coarsely equivalent to $\pi_{1}(K)=\Gamma$, we obtain by Theorem 3.6 and Proposition $4.8(1)$ that $\operatorname{asdim} \Gamma \geq \operatorname{asdim}_{\mathbf{Z}} \Gamma=\operatorname{asdim}_{\mathbf{Z}} \tilde{K} \geq$ $\mathrm{X}-\operatorname{dim}_{\mathbf{Z}} \tilde{K} \geq n$.

REMARK. The same argument works for the groups $\Gamma$ with finite $c d(\Gamma)$ and $B \Gamma$ having finite skeleton in each dimension.

We recall that a group $\Gamma$ is of the type $V F P$ if it admits a subgroup of finite index of the type FP [Br].

Corollary 4.11. $v c d \Gamma \leq$ asdim $\Gamma$ for groups $\Gamma$ of the type VFP.

Famous Stollings-Swan theorem implies the following. 
Corollary 4.12. Every group $\Gamma$ of type $V F P$ with asdim $\Gamma \leq 1$ is virtually free.

This result without the VFP restriction was proven independently by Januszkiewicz and Swiatkowski [JS] and Gentimis [G].

Theorem 4.13. Suppose that $\operatorname{asdim}_{\mathbf{Z}} \Gamma=\operatorname{asdim}_{\mathbf{Z}_{p}} \Gamma$ for a group $\Gamma$ of FL type with finite asymptotic dimension and some prime $p$. Then $\operatorname{asdim} \Gamma=c d \Gamma$.

Proof. By Theorem 4.8(2) and Theorem 3.6, X-dim $\mathbf{z}_{p} \Gamma=\operatorname{asdim}_{\mathbf{Z}} \Gamma=\operatorname{asdim} \Gamma$. By Theorem $4.9 c d \Gamma=\mathrm{X}-\operatorname{dim}_{\mathbf{Z}} \Gamma \geq \mathrm{X}-\operatorname{dim}_{\mathbf{z}_{p}} \Gamma=\operatorname{asdim} \Gamma$. By Proposition $4.10 \operatorname{asdim} \Gamma=$ $c d \Gamma$.

\section{$\S 5$ A Counterexample to the Asymptotic Analog of Morita's theorem}

The main idea of the construction can be demonstrated on the following example. Let $p, q$ be two mutually prime numbers and let $D$ be a 2-disc with two disjoint discs removed from its interior. Let $S_{1}^{1}$ and $S_{2}^{1}$ be the boundaries of the removed discs and let $S^{1}$ be the external boundary. We consider a free $\mathbf{Z}_{p}$ action on $S_{1}^{1}$ and a free $\mathbf{Z}_{q}$ action on $S_{2}^{1}$. Let $M$ denote the quotient space which is obtained from $D$ by factorization of $S_{1}^{1}$ and $S_{2}^{1}$ to the orbit spaces. Since the equation $m p+n q=1$ has a solution in integers, there is a retraction of $M$ to $S^{1}$. The degree of any such retraction restricted to the circle $S_{1}^{1} / \mathbf{Z}_{p}$ is $m$ and the degree of the restriction to $S_{2}^{1} / \mathbf{Z}_{q}$ is $n$ for some $m, n$ satisfying $m p+n q=1$. Thus, for large $p$ and $q$ these degrees have to be also large. This allows to construct a uniform complex $M$ that admits a retraction to the 'boundary' and such that every such retraction has the Lipschitz constant large.

Now we present the construction. Fix two primes $p>q^{2}$. For every natural $k$ we define a 2-dimensional complex $M_{k}$ as follows. Let $T_{p}$ be the mapping cylinder of the degree $p$ map $z^{p}: S^{1} \rightarrow S^{1}$. For a subdivisions of $S^{1}$ into $p^{i+1}$ and $p^{i}$ pieces we fix a mapping cylinder triangulation $T_{p}^{i}$ on $T_{p}$. Fixing orientation on $S^{1}$ we may assume that the complex $T_{p}^{i}$ is oriented. We consider the union $M_{k}^{p}=T_{p}^{k-1} \cup T_{p}^{k-2} \cup \cdots \cup T_{p}^{1}$ with identification of the image of $T_{p}^{i}$ with the domain of $T_{p}^{i-1}$. Let $s \in S^{1}$ be a base point. We may assume that it is taken by the map $z^{p}$ to the base point (consider $s=1$ ). Let $M_{k}^{\prime}=M_{k}^{p} \vee M_{k}^{q}$ be the wedge of these complexes with the base vertices located in the domains. Let $M$ be a mapping cylinder of the map $\phi: S^{1} \rightarrow S^{1} \vee S^{1}$ that collapses two points in $S^{1}$. We may supply $M$ with a triangulation having the following properties: (1) every vertex belongs to at most $p$ edges; (2) the domain $S^{1} \subset M$ of $\phi$ has 3 edges; (3) one circle in the target space $S^{1} \vee S^{1} \subset M$ of $\phi$ has $(3 p)^{k}$ edges and the other has $(3 q)^{k}$ edges. Glue $M$ along the target to $M_{k}^{\prime}$ to obtain $\tilde{M}_{k}$. Then we consider a 2 -simplex $\Delta$ subdivided in four 2-simplices by middle points of the edges and delete the interior of 
the middle simplex $D=\Delta \backslash$ Int $\sigma$. Glue $\tilde{M}_{k}$ to $\partial \sigma$ along the domain of $M$ to obtain $M_{k}$. We denote $\partial M_{k}=\partial \Delta \subset D$ and will refer to this set as to the boundary of $M_{k}$. The end circles of $M_{p}^{k}$ and $M_{q}^{k}$ we call the boundaries of $p$-hole and $q$-hole in $M_{k}$ respectively and denote them by $S_{p}^{1}, S_{q}^{1}$. Note that they are triangles, i.e., they have three edges. Assume that $M_{k}$ is given a metric of uniform simplicial complex. We fix a map $\phi_{k}: M_{k} \rightarrow \Delta$ which is simplicial with respect to the midpoint subdivision of $\Delta$ and such that the original vertices of $\Delta$ have exactly one preimage each. Clearly, this map is $1 / 2$-Lipschitz for the uniform metric on $M_{k}$

We define complexes $M_{k, k-1 \ldots, k-i}$ for $i=0, \ldots, k-1$ by induction on $i$. Assume that the simplicial complex $M_{k, k-1 \ldots, k-i}$ is already constructed. For every 2-simplex $\Delta \subset M_{k, k-1 \ldots, k-i}$ we delete its interior and glue instead a copy of $M_{k, k-1 \ldots, k-i-1}$ along the boundary $\partial M_{k, k-1 \ldots, k-i-1}$. This defines a map

$$
\phi_{k, \ldots, k-i-1}: M_{k, \ldots, k-i-1} \rightarrow M_{k, \ldots, k-i}
$$

which is $1 / 2$-Lipschitz. For $j>i$ we denote $\phi_{j}^{i}=\phi_{k, \ldots, j} \circ \phi_{k, \ldots, j-1} \circ \cdots \circ \phi_{k, \ldots, i}$.

Proposition 5.1. The following holds true:

(1) For every $k$ there is a retraction $r: M_{k} \rightarrow \partial M_{k}$.

(2) The Lipschitz constant of every retraction $r$ is greater than $q^{k}$.

(3) The Lipschitz constant of every map $f: M_{k} \rightarrow S^{1}$ having the restriction $\left.f\right|_{\partial M_{k}}$ with the nonzero degree $\operatorname{deg}\left(\left.f\right|_{\partial M_{k}}\right) \neq 0$ is greater than $q^{k}$. Moreover, $\operatorname{Lip}\left(\left.f\right|_{\partial M_{k} \cup S_{p}^{1} \cup S_{q}^{1}}\right) \geq q^{k}$.

(4) The Lipschitz constant of every map $f: M_{k, \ldots, i} \rightarrow S^{1}$ with the nonzero degree restriction $\operatorname{deg}\left(\left.f\right|_{\partial M_{k, \ldots, i}}\right) \neq 0$ is greater than $q^{i}$.

Here $S^{1}$ is given a metric of the boundary of the standard 2-simplex.

Proof. (1). Note that $M_{k}$ is homotopy equivalent to a 2-complex obtained from the wedge $S_{e}^{1} \vee S_{p}^{1} \vee S_{q}^{1}$ by attaching a 2-cell along the loop $\bar{e} a^{p^{k}} b^{q^{k}}$. Let $n$ and $m$ be such natural numbers that $n p^{k}+m q^{k}=1$. We consider maps $r_{p}: S_{p}^{1} \rightarrow S^{1}$ and $r_{q}: S_{q}^{1} \rightarrow S^{1}$ of degree $n$ and $m$ respectively. Then the map $i d_{S^{1}} \cup r_{p} \cup r_{q}: S_{e}^{1} \vee S_{p}^{1} \vee S_{q}^{1} \rightarrow S^{1}$ has an extension $r: M_{k} \rightarrow \partial M_{k}$ since the attaching map composed with it has the degree $0=-1+n p^{k}+m q^{k}$.

(2). Note that $\operatorname{Lip}(r) \geq \operatorname{Lip}\left(r_{q}\right) \geq|m| \geq(p / q)^{k}>q^{k}$.

(3). Let $d=\operatorname{deg}\left(\left.f\right|_{\partial M_{k}}\right)$ and let $d_{p}=\operatorname{deg}\left(\left.f\right|_{S_{p}^{1}}\right)$, and $d_{q}=\operatorname{deg}\left(\left.f\right|_{S_{q}^{1}}\right)$. Then $d_{p} p^{k}+$ $d_{q} q^{k}=d$. If one of the coefficients $d_{p}, d_{q}$ is zero, then $\operatorname{Lip}(f) \geq \operatorname{Lip}\left(\left.f\right|_{\partial M_{k}}\right) \geq d \geq q^{k}$. If both are nonzero, then they are divisible by $d$ and $d_{q} / d \geq q^{k}$ as above. Hence, $\operatorname{Lip}(f) \geq q^{k}$. 
(4). By induction on $k-i$. For $i=k$, the result is proven in (2). For $i<k$ we consider two cases. First we consider the case when the restriction of $f$ to one of the end circles in $M_{k, \ldots, i}$ has nonzero degree. Then the restriction of $f$ to a copy of $M_{i}$ satisfies the conditions of (3). Therefore, $\operatorname{Lip}\left(\left.f\right|_{M_{i}}\right) \geq q^{i}$.

Next we assume that $f$ has zero degree on every end circle of $M_{k, \ldots, 1}$. Then, $f$ defines a map $\tilde{f}: M_{k, \ldots, i+1} \rightarrow \partial \Delta^{2}$ which agrees with $f$ on the 1-dimensional skeleton of $M_{k, \ldots, i+1}^{(1)} \subset M_{k, \ldots, i}$. By the induction assumption $\operatorname{Lip}(\tilde{f}) \geq q^{i+1}$. Since $\operatorname{Lip}(\tilde{f})=$ $\operatorname{Lip}\left(\left.\tilde{f}\right|_{M_{k, \ldots, i+1}^{(1)}}\right)$, we obtain $\operatorname{Lip}(f) \geq q^{i+1}$.

Let $q_{k}: M_{k} \rightarrow \Delta$ be a simplicial approximation of $\phi_{k}: M_{k} \rightarrow \Delta$. Let $n_{k} \in \mathbf{N}$, denote by $\xi_{k}:\left[0, n_{k}\right] \rightarrow[0,1]$ the continuous map that collapses $\left[0, n_{k}-1\right]$ to 0 and maps $\left[n_{k}-1, n_{k}\right]$ isometrically onto $[0,1]$. Note that $\xi_{k}$ is a simplicial approximation of an orientation preserving homeomorphism $\left[0, n_{k}\right] \rightarrow[0,1]$ where $\left[0, n_{k}\right]$ is subdivided into the intervals of length one. Let $g_{k}=q_{k} \times \xi_{k}: M_{k} \times\left[0, n_{k}\right] \rightarrow \Delta \times[0,1]$.

Proposition 5.2. There is $\lambda>0$ such that for every $k$ there is $n_{k} \in \mathbf{N}$ and a $\lambda$ Lipschitz map $f_{k}: M_{k} \times\left[0, n_{k}\right] \rightarrow S^{2}$ such that $\left.f_{k}\right|_{\partial\left(M_{k} \times\left[0, n_{k}\right]\right)}=\left.g_{k}\right|_{\partial\left(M_{k} \times\left[0, n_{k}\right]\right)}$ where $\partial\left(M_{k} \times\left[0, n_{k}\right]\right)=\partial M_{k} \times\left[0, n_{k}\right] \cup M_{k} \times\left\{0, n_{k}\right\}, S^{2}=\partial\left(\Delta^{2} \times[0,1]\right)$ with the $l_{1}$-product metric.

Proof. Let $m_{k}=\min \left\{\|\gamma\| \mid \delta \gamma=q_{k}^{*}\left(1_{\Delta}\right)\right\}$ where $1_{\Delta} \in C^{2}(\Delta)$ is a simplicial cocycle that takes 1 on $\Delta$. According to Proposition 5.1(1) $m_{k}<\infty$. Assume that this $m_{k}$ is attained on a cochain $\gamma_{k}$. We take $n_{k}=m_{k}$ ! and consider the extension problem

$$
\begin{aligned}
\partial\left(M_{k}\right. & \left.\times\left[0, n_{k}\right]\right) \stackrel{g_{k} \mid}{\longrightarrow} \partial(\Delta \times[0,1]) \\
& \subset \downarrow \\
M_{k} & \times\left[0, n_{k}\right] .
\end{aligned}
$$

We note that the product $M_{k} \times\left[0, n_{k}\right]$ has a natural structure of a 3-dimensional cell complex where $\left[0, n_{k}\right]$ is subdivided into unit intervals, and the map $g_{k}$ takes its 2 -skeleton to $\partial(\Delta \times[0,1])$. Then the obstruction cocycle equals $c=g_{k}^{*}\left(1_{\Delta \times[0,1]}\right)$ where $1_{\Delta \times[0,1]}$ is the cellular 3-cocycle on $\Delta \times[0,1]$ that takes this 3-cell to one. We construct a 2-cochain $\beta_{k} \in C^{2}\left(M_{k} \times\left[0, n_{k}\right]\right)$ with $\delta \beta_{k}=c$ and with $\left|\beta_{k}\right| \leq 4$.

For every 1 -simplex $e$ in $M_{k}$ with $\gamma_{k}(e) \neq 0$ we set $\beta_{k}(e \times[i m, i m+1])=\operatorname{sgn}\left(\gamma_{k}(e)\right)$ for the integer $m=n_{k} /\left|\gamma_{k}(e)\right|$ and $i=0, \ldots,\left|\gamma_{k}(e)\right|-1$, and set $\beta_{k}(e \times[s, s+1])=0$ for all other cells. Here $\operatorname{sgn}(x)=\left\{\begin{array}{l}1 \text { if } x>0 \\ -1 \text { if } x<0 \\ 0 \text { if } x=0\end{array}\right.$ 
Note that $\beta_{k}\left(e \times\left[0, n_{k}\right]\right)=\gamma_{k}(e)$. Indeed,

$$
\beta_{k}\left(e \times\left[0, n_{k}\right]\right)=\sum_{i} \beta_{k}(e \times[i m, i m+1])=\left|\gamma_{k}(e)\right| \operatorname{sgn}\left(\gamma_{k}(e)\right)=\gamma_{k}(e) .
$$

For every 2-simplex $\sigma \subset M_{i}$ we set $\beta_{k}(\sigma \times\{l\})=-\beta_{k}(\partial \sigma \times[0, l])$ for all natural $l<n_{k}$ and we define $\beta_{k}(\sigma \times\{0\})=\beta_{k}\left(\sigma \times\left\{n_{k}\right\}\right)=0$.

Show that $\delta \beta_{k}=c=g_{k}^{*}\left(1_{\Delta \times[0,1]}\right)$. Indeed,

$$
\begin{gathered}
\delta \beta_{k}(\sigma \times[s-1, s])=\beta_{k}(\partial \sigma \times[s-1, s]+\sigma \times\{s\}-\sigma \times\{s-1\})=\beta_{k}(\partial \sigma \times[s-1, s])- \\
\beta_{k}(\partial \sigma \times[0, s])+\beta_{k}(\partial \sigma \times[0, s-1])=0=g_{k}^{*}\left(1_{\Delta \times[0,1]}\right)(\sigma \times[s-1, s])
\end{gathered}
$$

for $s<n_{k}$. For $s=n_{k}$ we obtain

$$
\begin{gathered}
\delta \beta_{k}(\sigma \times[s-1, s])=\beta_{k}(\partial \sigma \times[s-1, s])+\beta_{k}(\partial \sigma \times[0, s-1])=\beta_{k}\left(\partial \sigma \times\left[0, n_{k}\right]\right)=\gamma_{k}(\partial \sigma)= \\
\delta \gamma_{k}(\sigma)=q_{k}^{*}\left(1_{\Delta}\right)(\sigma)=\left(q_{k} \times \xi\right)^{*}\left(1_{\Delta \times[0,1]}\right)(\sigma \times[s-1, s]) .
\end{gathered}
$$

We show that the cochain $\beta_{k}$ is bounded. Let $\partial \sigma=a+b+c$ where $a, b, c$ are sides (with signs) of a 2-simplex $\sigma \subset M_{k}$. Note that

$$
\begin{gathered}
\left|\beta_{k}(\sigma \times\{l\})\right|=\left|\beta_{k}(\partial \sigma \times[0, l])\right|=\left|\beta_{k}(a \times[0, l])+\beta_{k}(b \times[0, l])+\beta_{k}(c \times[0, l])\right|= \\
\left|\operatorname{sgn}\left(\gamma_{k}(a)\right)\left[\frac{l\left|\gamma_{k}(a)\right|}{n_{k}}\right]+\operatorname{sign}\left(\gamma_{k}(b)\right)\left[\frac{l\left|\gamma_{k}(b)\right|}{n_{k}}\right]+\operatorname{sgn}\left(\gamma_{k}(c)\right)\left[\frac{l\left|\gamma_{k}(c)\right|}{n_{k}}\right]\right| \leq \\
\frac{l}{n_{k}}\left(\gamma_{k}(a)+\gamma_{k}(b)+\gamma_{k}(c)\right)+3=\leq \frac{l}{n_{k}}\left(q_{k}^{*}\left(1_{\Delta}\right)(\sigma)\right)+3 \leq 4
\end{gathered}
$$

where $[x]$ denote the integral part of $x$.

By the Obstruction Theory (see Proposition 3.4) the map $g_{k}$ restricted to the 2skeleton of $M_{k} \times\left[0, n_{k}\right]$ can be changed on 2-cells lying in $M_{k} \times\left[0, n_{k}\right] \backslash \partial\left(M_{k} \times\left[0, n_{k}\right]\right)$ without changing on the 1-skeleton by means of the cochain $\beta_{k}$ in such a way that a new map is $\mu\left(\left|\beta_{k}\right|\right)$-Lipschitz and it is extendible over $M_{k} \times\left[0, n_{k}\right]$ to a $\lambda$-Lipschitz map $f_{k}: M_{k} \times\left[0, n_{k}\right] \rightarrow S^{2}$. 
Theorem 5.3. There is a proper geodesic metric space $Y$ of bounded geometry with dimensions $\operatorname{asdim} Y=2$ and $\operatorname{asdim}(Y \times \mathbf{R})=2$.

Proof. Let $Y_{0}$ be the disjoint union of the uniform complexes $M_{k, \ldots, 1}$. To make it into a geodesic metric space we attach $M_{k, \ldots, 1}$ to the half-line $\mathbf{R}_{+}$at the point $2^{k} \in \mathbf{R}_{+}$for all $k$ :

$$
Y=\mathbf{R}_{+} \cup_{k} M_{k, \ldots, 1}
$$

In view of Theorem 3.6 it suffices to prove that $\operatorname{asdim} Y \geq 2$. We show that

$$
A H^{2}\left(\sqcup_{k} M_{k, \ldots, 1}, \sqcup_{k} \partial M_{k, \ldots, 1}\right) \neq 0 \text {. }
$$

We note that for every $k$ the projection $\phi_{k}^{i}$ takes $M_{k, \ldots, i}$ to $\Delta^{2}$ in such a way that it is a homeomorphism on the boundary $\partial M_{k, \ldots, i} \cong \partial \Delta^{2}$. Let $i: \mathbf{N}_{+} \rightarrow \mathbf{N}_{+}, i(k) \leq k$, $\lim i(k)=\infty$ and let $q_{i}: \sqcup_{k} M_{k, \ldots, i(k)} \rightarrow \sqcup_{k} \Delta^{2}$ be a simplicial approximation of $\sqcup_{k} \phi^{i}(k)_{k}$. We show that for every $i: \mathbf{N}_{+} \rightarrow \mathbf{N}_{+}, i(k) \leq k, \lim i(k)=\infty$,

$$
q_{i}^{*}: A H_{b}^{2}\left(\sqcup_{k} \Delta^{2}, \sqcup_{k} \partial \Delta^{2}\right) \rightarrow A H_{b}^{2}\left(\sqcup_{k} M_{k, \ldots, i(k)}, \sqcup_{k} \partial M_{k, \ldots, i(k)}\right)
$$

takes the fundamental class $\mu$ to nonzero element. Then

$$
A H_{b}^{2}\left(\sqcup_{k} \Delta^{2}, \sqcup_{k} \partial \Delta^{2}\right) \rightarrow A H_{b}^{2}\left(\sqcup_{k} M_{k, \ldots, 1}, \sqcup_{k} \partial M_{k, \ldots, 1}\right)
$$

will be a nonzero homomorphism.

Indeed, the image $q_{i}^{*}\left(1_{\Delta^{2}}\right)$ of the fundamental cocycle is the obstruction cocycle $C_{f}$ for a retraction $f: M_{k, \ldots, i(k)} \rightarrow \partial \Delta^{2}$ defined on the 1-skeleton as $q_{i}$. By Proposition 5.1(1) and the Obstruction Theory, $C_{f}=\delta \gamma_{k}$ for some simplicial cochain $\gamma_{k} \in C^{1}\left(M_{k, \ldots, i(k)}\right.$. By Proposition 5.1 (4) and the Obstruction Theory, $\left\|\gamma_{k}\right\| \geq q^{i}(k)-1$. Since $i(k) \rightarrow \infty$, the cocycle $q^{*}\left(1_{\Delta^{2}}\right)$ defines a nontrivial element in $A H_{b}^{2}\left(\sqcup_{k} M_{k, \ldots, i(k)}, \sqcup_{k} \partial M_{k, \ldots, i(k)}\right)$.

Now we show that $\operatorname{asdim}\left(Y_{0} \times \mathbf{R}\right) \leq 2$. We construct an anti-Čech approximation of $Y_{0} \times \mathbf{R}$ by 2 -dimensional complexes. We recall that the map $\phi_{j}^{i}: M_{k, \ldots, j} \rightarrow M_{k, \ldots, i}$ is defined for $k \geq j \geq i$. Let $Y_{i}=M_{i} \sqcup M_{i+1, i} \sqcup M_{i+2, i+1, i} \sqcup \ldots$ We denote

$$
\psi_{i}^{1}=\sqcup_{k \geq i} \phi_{i}^{1}: \sqcup_{k \geq i} M_{k, \ldots, 1}=Y_{0} \rightarrow Y_{i}
$$

and

$$
\psi_{i}=\phi_{i} \sqcup \phi_{i+1, i} \sqcup \phi_{i+2, i+1, i} \sqcup \cdots: Y_{i} \rightarrow \Delta \sqcup Y_{i+1}
$$


We consider the map $\psi_{i} \times \frac{1}{n_{i}}: Y_{i} \times \mathbf{R} \rightarrow\left(\Delta \sqcup Y_{i+1}\right) \times \mathbf{R}$ where $n_{i}$ is from Proposition 5.2. We subdivide $\mathbf{R}$ in the first product into the interval of the form $\left[\ln _{i}, l(l+1) n_{i}\right]$ and subdivide $\mathbf{R}$ in the second product into the unit intervals to turn $\psi_{i} \times \frac{1}{n_{i}}$ into a cellular map. By Proposition 5.2 there is a $\lambda$-Lipschitz sweeping $\xi_{i}: Y_{i} \times \mathbf{R} \rightarrow\left(\left(\Delta \sqcup Y_{i+1}\right) \times\right.$ $\mathbf{R})^{(2)}=N_{i}$ onto the 2-skeleton. Then the composition $\xi_{i} \circ\left(\psi_{i}^{1} \times \frac{1}{i}\right): Y_{0} \times \mathbf{R} \rightarrow N_{i}$ is a uniformly cobounded $\lambda / i$-Lipschitz map onto a 2-dimensional complex. To make it into a genuine anti-Čech approximation one needs to triangulate all the prisms in $N_{i}$.

Theorem 5.3 together with Proposition 4.2 implies

Corollary 5.4. If $Y$ as above, then $X-\operatorname{dim} Y=1$.

\section{$\S 6$ A CONSTRUCtion of LARGE Simplicial COMPLEXES}

We present here a construction of infinite locally finite uniform simplicial complexes. Let $\left\{\phi_{k}: M_{k} \rightarrow \Delta^{n}, \chi_{k}: M_{k} \rightarrow \Delta^{n}\right\}$ be a sequence of maps of $n$-dimensional simplicial complexes such that for each $k, \chi_{k}$ is a light simplicial map (and hence a retraction) and $\phi_{k}$ is a simplicial map to a some subdivision $\tau_{k}$ of the of $\Delta^{n}$ such that $\operatorname{mesh}\left(\tau_{k}\right)<\delta<1$ for fixed $\delta$ for all $k$. We recall that a map $\phi$ is called light if all point preimages are 0 dimensional. A light simplicial map $\phi: M \rightarrow \Delta^{n}$ is an isomorphism on every $n$-simplex $\sigma \subset M$. Williams calls the complexes that admit a light simplicial map onto the simplex $\Delta^{n}$ as complexes over $\Delta^{n}[\mathrm{Wi}]$.

Let $s_{k}: \Delta^{n} \rightarrow M_{k}$ be sections of $\chi_{k}$. 
Consider the following diagram:

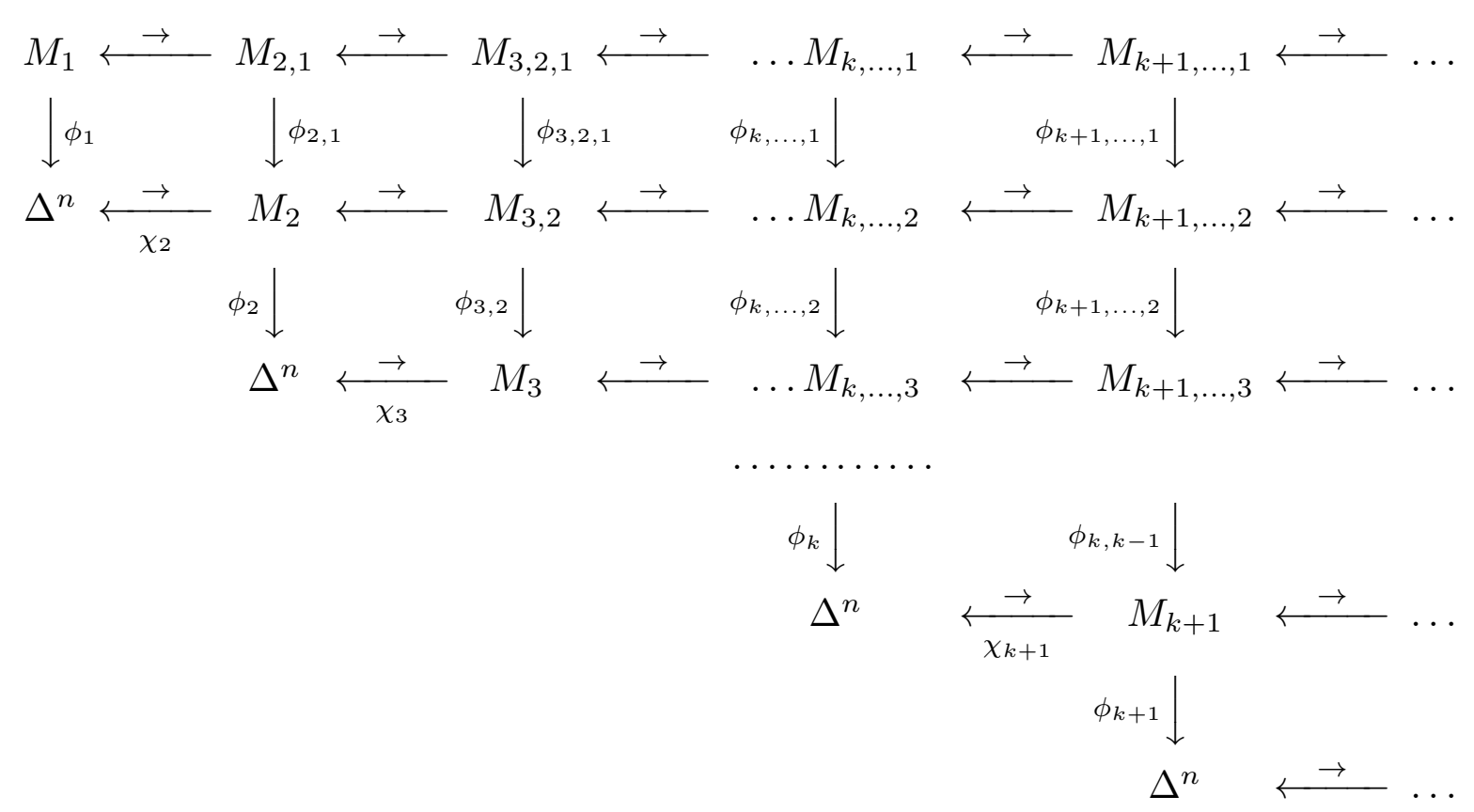

where all horizontal left arrows are generated by the maps $\chi_{k}$ by taking pull-back, all horizontal right arrows are generated by sections $s_{k}: \Delta^{n} \rightarrow M_{k}$ of maps $\chi_{k}$, and all squares are pull-back diagrams. We define simplicial complexes $Y_{k}$ as the direct limit of embeddings:

$$
Y_{k}=\lim _{\rightarrow}\left\{M_{k} \rightarrow M_{k+1, k} \rightarrow M_{k+2, k+1, k} \rightarrow \ldots\right\} .
$$

Note that for $k<l$ there are the natural projections $\phi_{l}^{k}: Y_{k} \rightarrow Y_{l}$ obtained as the direct limits in the above diagram. We denote the top horizontal sections by $\sigma_{k}: M_{k, \ldots, 1} \rightarrow$ $M_{k+1, \ldots, 1}$. Denote by

$$
Y=Y_{1}=\lim _{\rightarrow}\left\{M_{1} \stackrel{\sigma_{1}}{\longrightarrow} M_{2,1} \stackrel{\sigma_{2}}{\longrightarrow} M_{3,2,1} \rightarrow \ldots\right\} .
$$

We fix a simplicial approximation $\rho_{k}: \tau_{k} \rightarrow \Delta^{n}$ of the identity map $i d:\left|\tau_{k}\right| \rightarrow \Delta$. It defines a simplicial approximation $q_{k}^{k-1}$ of the map $\phi_{k}^{k-1}$.

We assume that all $Y_{k}$ are given the uniform geodesic metrics.

Proposition 6.1. The family

$$
\left\{\phi_{k}^{1}: Y \rightarrow Y_{k}, q_{k+1}^{k}, \phi_{k+1}^{k}\right\}
$$


is a regular anti-Čech approximation of $Y$.

Proof. We consider $\mathcal{U}_{k}=\left(\phi_{k}^{1}\right)^{-1}\left\{O s t\left(v, Y_{k}\right)\right\}$. By the construction $\phi_{k}^{1}$ is a simplicial map to a $k$-1-iterated $\delta$-subdivision of a uniform complex. Therefore, $\phi_{k}^{1}$ is $\delta^{k-1}$-Lipschitz on every simplex of $Y$. Since the metric on $Y$ is geodesic, it is $\delta^{k-1}$-Lipschitz. Since $\delta^{k} \rightarrow 0$, the Lebesgue numbers $L\left(\mathcal{U}_{k}\right)$ tends to infinity. Since $\phi_{k+1}^{k}$ is simplicial with respect to a subdivision, for every vertex $v \in Y_{k}$ there is a vertex $u \in Y_{k+1}$ such that

$$
\phi_{k+1}^{k}\left(O s t\left(v, Y_{k}\right)\right) \subset \operatorname{Ost}\left(\phi_{k+1}^{k}(v), \tau_{k+1}\right) \subset O s t\left(u, Y_{k+1}\right) .
$$

Therefore, $O s t\left(v, Y_{k}\right) \subset\left(\phi_{k+1}^{k}\right)^{-1}\left(O s t\left(u, Y_{k+1}\right)\right.$ and hence

$$
\left(\phi_{k}^{1}\right)^{-1}\left(O s t ( v , Y _ { k } ) \subset ( \phi _ { k + 1 } ^ { 1 } ) ^ { - 1 } \left(O s t\left(u, Y_{k+1}\right)\right.\right.
$$

and the condition $\mathcal{U}_{k} \prec \mathcal{U}_{k+1}$ is checked.

REMARK. The space $Y$ has natural compactification

$$
\bar{Y}=\lim _{\leftarrow}\left\{M_{1} \leftarrow M_{2,1} \leftarrow M_{3,2,1} \leftarrow \ldots\right\}
$$

The complexes $M_{k}$ constructed in $\S 5$ can be considered to be oriented. Let $\chi: M_{k} \rightarrow$ $\Delta^{2}$ be a simplicial map defined by the orientation. We consider the following triangulation $\tau$ of the 2-simplex $\Delta^{2}$ : First, we take the midpoint subdivision of $\Delta^{2}$ and then take the cone subdivision of the central 2-simplex. Let $\phi_{k}:\left(M_{k}, S^{1}\right) \rightarrow\left(\Delta^{2}, \partial \Delta^{2}\right)$ be a simplicial map to the subdivision $\tau$ of $\Delta^{2}$ which takes $M_{k}^{\prime}$ to the center and maps $D$ to $D$ by the identity map. Then Theorem 5.3 can be stated as follows.

Theorem 6.2. The family $\left\{\phi_{k}: M_{k} \rightarrow \Delta^{2}, \chi_{k}: M_{k} \rightarrow \Delta^{2}\right\}$ for any choice of sections $s_{k}: \Delta^{2} \rightarrow M_{k}$ defines the space $Y$ as above with an anti-ČCh approximation $\left\{\phi_{k}^{1}: Y \rightarrow\right.$ $\left.Y_{k}\right\}$ such that $\operatorname{asdim}(Y \times \mathbf{R})=\operatorname{asdim} Y=2$.

Note that if $s_{k}\left(\Delta^{2}\right) \cap \partial M_{k}=\emptyset$ for all $k$ then the boundary of the complex $Y$ is homeomorphic to the circle $S^{1}$.

\section{REFERENCES}

[Ba] A. Bartels, Squeezing and higher algebraic K-theory, K-theory 28 (2003), 19-37.

[BD1] G. Bell and A. Dranishnikov, On asymptotic dimension of groups acting on trees, Geom. Dedicata 103 (2004), 89-101.

[BD2] G.Bell and A. Dranishnikov, A Hurewicz-type theorem for asymptotic dimension and applications to geometric group theory., Trans AMS (to appear). 
[Br] K. Brown, Cohomology of groups (1982), Springer-Verlag.

[CG] G. Carlsson and B. Goldfarb, The integral K-theoretic Novikov conjecture for groups with finite asymptotic dimension, Inventiones Math. 157 No 2 (2004), 405-418.

[Dr1] A. Dranishnikov, Asymptotic topology, Russian Math. Surveys 55:6 (2000), 71-116.

[Dr2] A. Dranishnikov, On hypersphericity of manifolds with finite asymptotic dimension, Trans. Amer. Math. Soc. 355 no 1 (2003), 155-167.

[Dr3] A. Dranishnikov, Cohomological dimension theory, Topology Atlas.

[Dr4] A. Dranishnikov, On Hypereuclidean manifolds, Geom. Dedicata (to appear).

[DFW] A. Dranishnikov, S. Ferry, S. Weinberger, An etale approach to the Novikov conjecture, Preprint of MPI für Mathematik (2005).

[DS] A. Dranishnikov J. Smith, Asymptotic dimension of discrete groups, Fundamenta Mathematicae 189 (2006), 27-34.

[G] A. Gentimis, Asymptotic dimension of finitely presented groups, Preprint (2005).

[Gr1] M. Gromov, Asymptotic invariants of infinite groups, Geometric Group Theory, vol 2, Cambridge University Press, 1993.

[Gr2] M. Gromov, Random walk on random groups, GAFA 13:1 (2003), 73-146.

[Ha] A. Harlap, Local homology and cohomology, homological dimension, and generalized manifolds., Mat. Sbornik 96 (138) (1975), 347-373.

[HR] N. Higson and J. Roe, Amenable group actions and the Novikov conjecture., J. Reine Angew. Math. 519 (2000), 143-153.

[Hu] S.T. Hu, Homotopy theory, Academic Press, 1959.

[JS] T. Januszkiewicz and J. Swiatkowski, Filling invariants in systolic complexes and groups, Preprint (2005).

[Ro1] J. Roe, Coarse cohomology and index theory for complete Riemannian manifolds, Memoirs Amer. Math. Soc. No. 497, 1993.

[Ro2] J. Roe, Lectures on coarse geometry, University Lecture series, Volume 31 (2003), AMS.

[Wi] R.F. Williams, A useful functor and three famous examples in topology, Trans. Amer. Math. Soc. 106 (1963), 319-329.

[Yu G. Yu, The Novikov conjecture for groups with finite asymptotic dimension, Ann. of Math 147 (1998), no. 2, 325-355.

University of Florida, Department of Mathematics, P.O. Box 118105, 358 Little Hall, Gainesville, FL 32611-8105, USA

E-mail address: dranish@math.ufl.edu 